\title{
An overview of the SOLVE/THESEO 2000 campaign
}

\author{
Paul A. Newman, ${ }^{1}$ Neil R. P. Harris, ${ }^{2}$ Alberto Adriani, ${ }^{3}$ Georgios T. Amanatidis, ${ }^{4}$ \\ James G. Anderson, ${ }^{5}$ Geir O. Braathen, ${ }^{6}$ William H. Brune, ${ }^{7}$ Kenneth S. Carslaw, ${ }^{8}$ \\ Michael S. Craig, ${ }^{9}$ Philip L. DeCola,${ }^{10}$ Marielle Guirlet, ${ }^{11}$ R. Stephen Hipskind, ${ }^{9}$ \\ Michael J. Kurylo, ${ }^{10,12}$ Harry Küllmann, ${ }^{13}$ Niels Larsen, ${ }^{14}$ Gérard J. Mégie, ${ }^{15}$ \\ Jean-Pierre Pommereau, ${ }^{16}$ Lamont R. Poole, ${ }^{17}$ Mark R. Schoeberl, ${ }^{1}$ Fred Stroh, ${ }^{18}$ \\ Owen B. Toon, ${ }^{19}$ Charles R. Trepte, ${ }^{17}$ and Michel Van Roozendael ${ }^{20}$ \\ Received 18 September 2001; revised 2 November 2001; accepted 9 November 2001; published 25 October 2002.
}

[1] Between November 1999 and April 2000, two major field experiments, the Stratospheric Aerosol and Gas Experiment (SAGE) III Ozone Loss and Validation Experiment (SOLVE) and the Third European Stratospheric Experiment on Ozone (THESEO 2000), collaborated to form the largest field campaign yet mounted to study Arctic ozone loss. This international campaign involved more than 500 scientists from over 20 countries. These scientists made measurements across the high and middle latitudes of the Northern Hemisphere. The main scientific aims of SOLVE/THESEO 2000 were to study (1) the processes leading to ozone loss in the Arctic vortex and (2) the effect on ozone amounts over northern midlatitudes. The campaign included satellites, research balloons, six aircraft, ground stations, and scores of ozonesondes. Campaign activities were principally conducted in three intensive measurement phases centered on early December 1999, late January 2000, and early March 2000. Observations made during the campaign showed that temperatures were below normal in the polar lower stratosphere over the course of the 1999-2000 winter. Because of these low temperatures, extensive polar stratospheric clouds (PSC) formed across the Arctic. Large particles containing nitric acid trihydrate were observed for the first time, showing that denitrification can occur without the formation of ice particles. Heterogeneous chemical reactions on the surfaces of the PSC particles produced high levels of reactive chlorine within the polar vortex by early January. This reactive chlorine catalytically destroyed about $60 \%$ of the ozone in a layer near $20 \mathrm{~km}$ between late January and mid-March 2000, with good agreement being found between a number of empirical and modeling studies. The measurements made during SOLVE/THESEO 2000 have improved our understanding of key photochemical parameters and the evolution of ozone-destroying forms of chlorine. INDEX TERMS: 0342 Atmospheric Composition and Structure: Middle atmosphere - energy deposition; 3334 Meteorology and Atmospheric Dynamics: Middle atmosphere dynamics (0341, 0342); KEYWORDS: SOLVE, THESEO 2000, ozone, ozone loss, stratosphere, polar vortex

Citation: Newman, P. A., et al., An overview of the SOLVE/THESEO 2000 campaign, J. Geophys. Res., 107(D20), 8259, doi:10.1029/2001JD001303, 2002.

\section{Introduction}

\subsection{Background}

[2] Between November 1999 and April 2000, two major field experiments, the NASA-sponsored SAGE III Ozone

\footnotetext{
${ }^{1}$ NASA Goddard Space Flight Center, Greenbelt, Maryland, USA.

${ }^{2}$ European Ozone Research Coordinating Unit, Cambridge, UK.

${ }^{3}$ Consiglio Nazionale delle Ricerche, ISAC, Rome, Italy.

${ }^{4}$ European Commission, Research DG, Brussels, Belgium.

${ }^{5}$ Harvard University, Cambridge, Massachusetts, USA

${ }^{6}$ Norsk Institutt for Luftforskning, Kjeller, Norway.

${ }^{7}$ Pennsylvania State University, University Park, Pennsylvania, USA.

${ }^{8}$ University of Leeds, Leeds, UK.

${ }^{9}$ NASA Ames Research Center, Moffett Field, California, USA.

${ }^{10}$ NASA Headquarters, Washington D.C., USA.
}

Loss and Validation Experiment (SOLVE) and the European Commission-led Third European Stratospheric Experiment on Ozone (THESEO 2000), collaborated to form the largest field campaign yet mounted to study Arctic ozone loss. The main scientific aims of the combined campaign were to

\footnotetext{
${ }^{11}$ ACRI-ST, Sophia-Antipolis, France.

${ }^{12}$ National Institute of Standards and Technology, Gaithersburg, Maryland, USA.

${ }^{13}$ University of Bremen, Bremen, Germany.

${ }^{14}$ Danish Meteorological Institute, Copenhagen, Denmark.

${ }^{15}$ Service d'Aéronomie, Institut Pierre Simon Laplace, Paris, France.

${ }^{16}$ Centre National de la Recherche Scientifique, SA, Verrières le Buisson, France.

${ }^{17}$ NASA Langley Research Center, Hampton, Virginia, USA.

${ }^{18}$ Forschungszentrum Jülich, Jülich, Germany.

${ }^{19}$ University of Colorado, Boulder, Colorado, USA

${ }^{20}$ Belgian Institute for Space and Aeronomy, Brussels, Belgium.
} 
study (1) the processes leading to ozone loss in the Arctic vortex and (2) the effect on ozone amounts over northern midlatitudes. A further aim was to validate measurements from the third Stratospheric Aerosol and Gas Experiment (SAGE III) instrument. Unfortunately, SAGE III was not launched on schedule, and so this goal was not directly achieved. However, measurements from several satellite instruments are being used in conjunction with SOLVE/ THESEO 2000 data for scientific and validation studies. SOLVE/THESEO 2000 involved research aircraft, balloons, ozonesondes, and ground-based and satellite instruments, which were augmented by meteorological and chemical models. In all, more than 500 scientists from over 20 countries (the European Union (EU), Canada, Iceland, Japan, Norway, Poland, Russia, Switzerland, and the United States) were involved. Descriptions of the SOLVE/THESEO 2000 activities and the early findings were published in the Stratospheric Processes and Their Role in Climate (SPARC) newsletter [Newman, 2000; Harris et al., 2001].

[3] The joint SOLVE/THESEO 2000 campaign represents a new level of cooperation between researchers and agencies from the EU, the U.S., and other countries. This cooperation was officially implemented under the 1998 "European Union/United States Science and Technology Cooperation Agreement," which was crafted to promote, develop, and facilitate cooperative research and development activities for mutual benefit in virtually all areas of natural sciences and engineering. In this light, the THESEO 2000 Core Group and the SOLVE Management Team recognized that the issues of chemical ozone loss and the expected ozone recovery in a changing atmosphere (containing increased abundances of greenhouse gases) are complex and that their study would require scientific research activities that are facilitated by international collaboration. Following early discussions, the two planning groups held a joint meeting in November 1999 and decided to join efforts in the close coordination and implementation of the two campaigns. Common data protocols, data dissemination policies, and a joint workshop on the results were also arranged. There were international suites of instruments on aircraft and balloons. The success of this approach is reflected in the number of papers with authors from Europe and the United States.

[4] Arctic ozone loss has been extensively studied since the discovery of the Antarctic ozone hole [Farman et al., 1985]. The existence of this large area of depleted ozone immediately raised the question of whether a similar phenomenon could occur over the Arctic, closer to the heavily populated areas of Asia, Europe, and North America. The confirmation that the ozone loss was caused by chlorine and bromine chemistry underlined the importance of the question. As a result, a number of large scientific campaigns have investigated ozone loss in the Arctic stratosphere during winter and spring. These campaigns include the following: (1) Airborne Arctic Stratospheric Experiment (AASE, 1989); (2) European Arctic Stratospheric Ozone Experiment (EASOE, 1991-1992); (3) Second Airborne Arctic Stratospheric Experiment (AASE II, 1991-1992); (4) Second European Stratospheric Arctic and Midlatitude Experiment (SESAME, 1994-1995); (5) Photochemistry of Ozone Loss in the Arctic Region in Summer (POLARIS, 1997); and (6) Third European Stratospheric Experiment on Ozone (THESEO, 1998-1999).
[5] There have also been a number of smaller, focused campaigns investigating processes such as the Vortex Ozone Transport Experiment (VOTE, 1996), the Polar Stratospheric Aerosol Experiment (POLSTAR, 1997), and Airborne Polar Experiment (APE-POLECAT, 1997). These campaigns have been complemented by longer-term observations from ground stations (e.g., the stratospheric composition measurements made within the Network for the Detection of Stratospheric Change (NDSC) and the ozone and meteorological observations made within the World Meteorological Organization-Global Atmosphere Watch (WMO-GAW) network) and by a number of satellite instruments (e.g., see the satellite section 2.2.1). Such continuity of observations is important, since a striking feature of Arctic ozone loss has been its large interannual variability. Multiyear records give the perspective with which to interpret results from individual, intensively studied winters.

[6] The processes leading to Arctic ozone loss have been closely studied, and qualitatively, they are fairly well understood. During October and November, a westerly circulation develops in the stratosphere as the polar stratosphere cools. This circumpolar vortex is the dominant feature of the stratospheric circulation during the Arctic winter and is the counterpart of the more stable, stronger, and long-lived Antarctic vortex. During the long periods of darkness at high latitudes in winter, as the Arctic vortex cools radiatively, sufficiently low temperatures (195 K or below) can be reached at which polar stratospheric clouds (PSC) can form. Heterogeneous chemical reactions occur on the surface of the PSC particles. These reactions lead to the conversion of relatively inactive chlorine compounds such as $\mathrm{HCl}$ and $\mathrm{ClONO}_{2}$ into $\mathrm{ClO}$, and this $\mathrm{ClO}$ causes rapid chemical destruction of ozone in the presence of sunlight. This catalytic ozone loss is deactivated by the reformation of $\mathrm{ClONO}_{2}$ and $\mathrm{HCl}$. These processes largely take place in the Arctic vortex. Then, as the vortex circulation breaks down in spring, ozone-depleted air is mixed with midlatitude air, extending the influence of Arctic ozone loss to lower latitudes.

[7] Levels of chlorine and bromine in the Arctic are high enough to cause rapid ozone losses provided the meteorological conditions are cold enough for the formation of PSCs, particularly during late February and March, when sunlight returns to the vortex region. There are large interannual variabilities in the dynamics and temperature of the Arctic stratosphere, and large ozone losses have occurred in the colder winters but not in the warmer ones. Modeling studies suggest that changes in climate will additionally cool the stratosphere, potentially leading to enhanced PSC formation and a longer-lived Arctic vortex [Shindell et al., 1998]. These mechanisms would increase the frequency of winters with large Arctic ozone losses. An improved understanding of the dynamic, radiative, and chemical processes involved is required to increase confidence in model studies of the possible future atmosphere.

[8] The main results of SOLVE/THESEO 2000 are being published in a series of special sections of the Journal of Geophysical Research. In this paper we present an overview of the campaign. In the rest of the introduction, the scientific goals of the campaign are described. In section 2, the full scope of the mission is described with details about the timing and coverage, the platforms (aircraft, balloons, ground-based stations and satellites) and instruments involved, and the 
meteorological and modeling support activities. A brief outline of the main results to date is given in section 3 .

\subsection{Scientific Goals}

[9] Each element of the chain of processes involved in chemical ozone loss needs to be understood quantitatively if the future evolution of ozone over the Arctic and the northern midlatitudes is to be accurately predicted. Based on the simple picture given above, the scientific aims can be divided into four main categories: (1) transport and dynamics, (2) formation and composition of PSCs, (3) improved quantification of the activation and deactivation of halogen oxides, and (4) empirical and modeled calculations of chemical ozone loss. These more detailed aims are now considered in turn.

\subsubsection{Transport and Dynamics}

[10] Understanding the transport and dynamics of the development and breakup of the Arctic vortex, needed to fully constrain the chemical evolution, requires precise large-scale observations over at least one Arctic winter. In situ observations of the Arctic stratosphere during the early and middle winter have been relatively sparse. It was important to study this period in detail because the dynamical evolution of the vortex during this period has a major significance in terms of the chemical and dynamical behavior in the January to March period, when nearly all the ozone loss takes place. For example, the amount of descent in the vortex resulting from radiative cooling determines the amounts of $\mathrm{Cl}_{y}, \mathrm{Br}_{y}$, and $\mathrm{NO}_{y}$ in spring, while the temperature history determines the extent of PSC formation and, indirectly, the degree of denitrification.

[11] The exchange of air between the polar vortex and the midlatitudes is also an important factor in the polar ozone budget. Further, the transport of low-ozone air from the polar vortex into midlatitudes can reduce midlatitude ozone levels. In addition, while midlatitude air is not easily transported into the polar vortex, occasionally, under disturbed stratospheric weather conditions, midlatitude air can be entrained into the vortex, significantly changing the vortex's chemical composition. Small-scale exchange can also provide weak transport across the vortex edge.

\subsubsection{Formation and Composition of PSCs}

[12] PSCs provide the surfaces for the heterogeneous reactions that initiate ozone loss, and they also sequester and redistribute (or remove) nitric acid and water from the stratosphere. The heterogeneous reaction rates on PSCs are known from laboratory measurements but are rather poorly constrained by atmospheric observations. A wide number of questions are still open with respect to the types of PSCs and their composition. These questions were principally addressed during SOLVE/THESEO 2000 by direct measurement and analysis of the chemical and physical composition of PSCs. Information was needed on the temporal evolution of PSCs, in particular, the nucleation of these particles and the evolution of their size distributions. Other important questions for PSCs concerned the spatial and horizontal morphologies of the PSC types and size distributions and their relation to the broad temperature fields. A good understanding of these factors is necessary for broadscale models to include valid parameterizations for PSC occurrence and the subsequent heterogeneous activation and denitrification process.

\subsubsection{Improved Quantification of the Activation and Deactivation of Halogen Oxides}

[13] The chemical depletion of ozone by halogen oxides involves three main areas: the activation processes, the ozone loss cycles, and the deactivation back to the reservoir species. The activation requires the presence of PSCs, and so an important question is how the seasonal evolution of the PSCs regulates the activation through the winter. Another important issue is how the chemical budgets of the nitrogen, chlorine, and bromine families evolve over the course of the winter owing to repeated activation events. In particular, the impact of synoptic-scale and mountain wave mesoscale temperature perturbations has to be considered for an understanding of chlorine activation.

[14] In terms of the ozone loss cycles, the questions mainly relate to understanding and improved quantification of the relative contributions among catalytic loss cycles to the overall ozone loss in the winter to spring period. In particular, what are the absolute rates of the catalytic cycles, and what is the relative importance of chlorine and bromine? Underlying these issues is how the different chemical families interact under a wide range of conditions and how this affects the overall chemical ozone loss.

[15] Finally, it is believed that deactivation (the termination of the halogen catalyzed ozone loss) depends on the reintroduction of $\mathrm{NO}_{2}$ into the gas phase through the photolysis of nitric acid, where it reacts with $\mathrm{ClO}$ to form the relatively long-lived $\mathrm{ClONO}_{2}$, and the rate of formation of $\mathrm{HCl}$. Key chemistry questions concern the rate of production of $\mathrm{NO}_{2}$ and $\mathrm{HCl}$ in spring. A critical issue is how chlorine deactivation processes, and hence ozone loss, depend on denitrification.

\subsubsection{Empirical and Modeled Calculations of Chemical Ozone Loss}

[16] A number of techniques for empirically estimating chemical ozone loss rates in the Arctic vortex have been developed in recent years. Several of these techniques were used during the $1999 / 2000$ winter. It is important to examine the internal consistency of these empirical estimates, as there has been considerable discussion of discrepancies between results from different winters.

[17] Comparison of these empirical estimates of ozone loss with the losses calculated by models was another priority for SOLVE/THESEO 2000. Several studies have shown significant disagreements between the modeled and empirical estimates [e.g., Becker et al., 2000; KilbaneDawe et al., 2001; Woyke et al., 1999], and these disagreements limit the confidence that can be placed in predictions of future losses. A particular puzzle has been the high ozone loss rates observed at the end of January in several years [e.g., Rex et al., 1999; Woyke et al., 1999]. Thorough comparisons using a range of models are needed to understand better the conditions where these disagreements arise.

\section{Mission Scope}

[18] The SOLVE/THESEO 2000 campaign was conducted primarily in three phases over the Arctic and midlatitude region during the winter of 1999-2000. Most activities took place at Kiruna, Sweden $\left(68^{\circ} \mathrm{N}, 21^{\circ} \mathrm{E}\right)$ or the Swedish Space Corporation (SSC) Esrange facility 40 


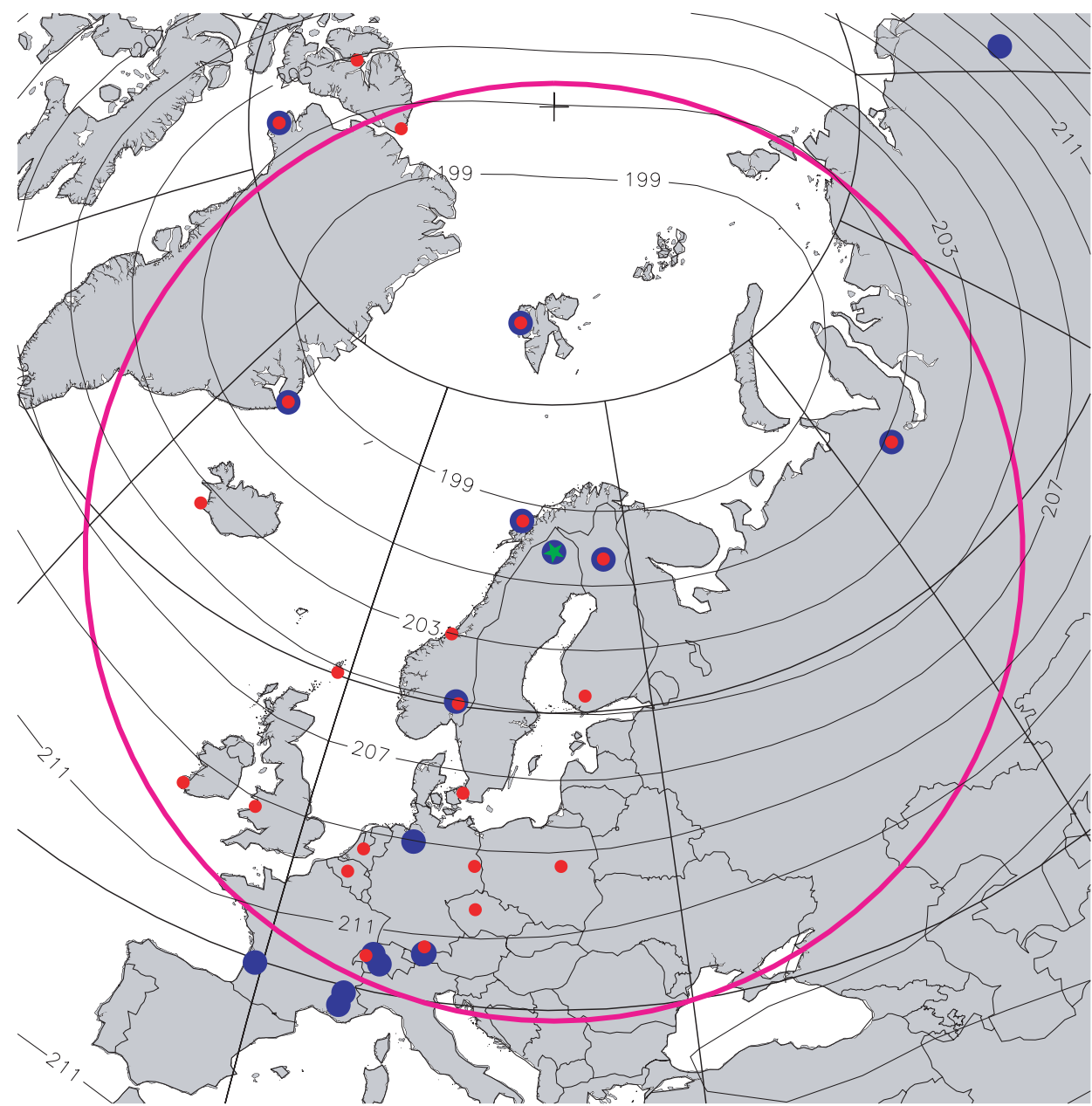

Figure 1. SOLVE/THESEO 2000 operations region, with Kiruna, Sweden, noted at the center of the image with the green star. The magenta line indicates a distance of $2700 \mathrm{~km}$ from Kiruna. The blue dots indicate ground observation sites, while the smaller red dots indicate ozonesonde sites. Also superimposed are 50-hPa January average temperature contours (1979-1999). On average, the coldest region of the lower stratosphere is centered on Spitzbergen.

$\mathrm{km}$ from Kiruna. In late 1999, during the first phase, the NASA DC-8 was deployed in Kiruna and two large and two small balloons were launched. Between mid-January and March 2000, in the second and third phases, 7 large balloons, 14 small balloons and 3 long-duration balloons carrying scientific payloads were launched successfully from Esrange. Two of the small balloons were launched from the Norwegian station in Andøya. Aircraft measurements in the Arctic region were made on board the NASA ER-2, NASA DC-8, DLR Falcon, the Centre National de la Recherche Scientifique/Institut National des Sciences de l'Univers (CNRS/INSU) ARAT, and the Swiss Air Force Lear jet. In addition, the CNRS/INSU Mystère 20 was flown at midlatitudes. There were simultaneous groundbased activities from the Scandinavian stations (ALOMAR, Kiruna, Ny-Ålesund, Sodankylä, Harestua) throughout the winter, along with other ground measurements from numerous sites across the Arctic (see Figure 1). In coordinated activities, the aircraft often flew upwind and downwind of balloon flight paths. Additional flights of ozonesondes were conducted at a number of sites across the Northern Hemisphere [Rex et al., 2002], and ground measurements were made at a number of Arctic and midlatitude sites.
[19] The Total Ozone Mapping Spectrometer (TOMS), Global Ozone Monitoring Experiment (GOME), and Polar Ozone and Aerosol Measurement (POAM) satellite instruments measured in sunlit regions of the stratosphere through the winter. Meteorological support was provided by several meteorological organizations, some with representatives in the field (FU-Berlin, U.S. National Oceanic and Atmospheric Administration/National Centers for Environmental Prediction (NOAA/NCEP), U.S. Naval Research Laboratory, NASA Goddard Space Flight Center (GSFC) Data Assimilation Office) to assist daily planning, and others through the provision of data (European Center for Medium-Range Weather Forecasts (ECMWF), Météo-France, Swedish Meteorological and Hydrological Institute (SMHI), UK Met Office (UKMO), and Deutscher Wetterdienst (DWD)). Modeling and theory support groups were essential for both the premission planning and the daily operational planning.

\subsection{Location and Timing}

[20] The SOLVE/THESEO 2000 campaign was designed for both extensive spatial coverage and fall-to-spring temporal coverage. In particular, the campaign was partitioned into three intensive measurement phases. The first phase 
Table 1. Satellite List

\begin{tabular}{|c|c|c|c|c|}
\hline Instrument & Satellite & Investigator & Institution & Measurements \\
\hline POAM III & French Spot 4 & R. Bevilacqua & Naval Res. Lab. & $\begin{array}{l}\text { profiles: } \mathrm{O}_{3}, \mathrm{H}_{2} \mathrm{O}, \mathrm{NO}_{2} \text {, aerosol } \\
\text { extinction }\end{array}$ \\
\hline TOMS & Earth Probe & R. McPeters & NASA/GSFC & column: $\mathrm{O}_{3}$ \\
\hline GOME & ERS 2 & J. Burro & Univ. of Bremen & $\begin{array}{l}\text { column: } \mathrm{O}_{3}, \mathrm{BrO}, \mathrm{NO}_{2}, \mathrm{OClO}, \mathrm{H}_{2} \mathrm{CO} \\
\text { profiles: } \mathrm{O}_{3}\end{array}$ \\
\hline HALOE & UARS & J. M. Russell & Hampton Univ. & $\begin{array}{l}\text { profiles: } \mathrm{O}_{3}, \mathrm{H}_{2} \mathrm{O}, \mathrm{NO}_{2}, \mathrm{~T}, \mathrm{HCl}, \mathrm{CH}_{4} \text {, } \\
\quad \mathrm{HF}, \mathrm{NO} \text {, aerosol extinction }\end{array}$ \\
\hline SAGE II & ERBS & & NASA/LRC & $\begin{array}{l}\text { profiles: } \mathrm{O}_{3}, \mathrm{H}_{2} \mathrm{O}, \mathrm{NO}_{2} \text {, aerosol } \\
\quad \text { extinction }\end{array}$ \\
\hline MLS & UARS & J. Waters & JPL & profiles: $\mathrm{O}_{3}, \mathrm{HNO}_{3}, \mathrm{ClO}$ \\
\hline
\end{tabular}

was set in late November and early December 1999, when the polar vortex typically forms and temperatures decrease below about $195 \mathrm{~K}$ (the nitric acid trihydrate saturation temperature) over wide regions. The second phase was chosen during the period when polar lower stratospheric temperatures usually reach their minimums and the vortex is strongest. Because this phase was timed to occur during the coldest period, formation of PSCs was expected to lead to extensive conversion of chlorine into reactive forms. The final phase of SOLVE/THESEO 2000 was timed to the recovery period of the polar vortex as temperatures rose above $195 \mathrm{~K}$, the vortex decreased in strength, and perturbed chemistry began to recover.

[21] The science requirements prescribed a need for regular and deep penetrations of the polar vortex along with good proximity to the coldest portion of the polar vortex. Kiruna, Sweden, provided an ideal site for SOLVE/THESEO 2000's principal activities. The Arena Arctica hangar at the Kiruna Airport was built in 1992 as a facility for accommodating large aircraft field projects on environmental issues. In late January, this hangar simultaneously accommodated the NASA ER-2, NASA DC-8, and DLR Falcon. A second hangar was available for use by the ARAT and Lear jet. The Arena Arctica also includes offices, laboratories, and shared rooms such as a pantry, a meeting room, dressing rooms, and workshops. The laboratory and office space on either side of the hangar housed all of the investigators and instruments for the aircraft portion of the campaign.

[22] Balloon operations were conducted at the Swedish Space Corporation's Esrange facility, which is approximately $40 \mathrm{~km}$ from Kiruna. This facility is able to launch balloons of up to $10^{6} \mathrm{~m}^{3}$ and includes a large $400 \mathrm{~m}$ by $250 \mathrm{~m}$ launch pad, a balloon tracking and control facility, and several laboratories for payload preparation. In addition, Esrange includes accommodation and dining facilities to handle the large teams of scientists associated with the balloons. A second balloon facility, where $10^{4} \mathrm{~m}^{3}$ balloons were launched, was at the Norwegian Rocket Range at Andøya.

[23] SOLVE/THESEO 2000 operations extensively covered the Arctic region, as displayed in Figure 1. The magenta line indicates the $2700-\mathrm{km}$ radius from Kiruna, roughly the operations zone for the aircraft. Kiruna is located at the center of the image by the green star. Ozonesonde launch sites were widely distributed across the polar region (as indicated by the red points in Figure 1). In addition, groundbased instruments were located at a number of sites across the polar and midlatitude region in support of SOLVE/ THESEO 2000. The observational sites in the EU project Compilation of Atmospheric Observations in Support of Satellite Measurements Over Europe (COSE; coordinator,
M de Mazière, Belgian Institute for Space Aeronomy (BISA)) are shown as the blue points in Figure 1.

\subsection{Platforms}

[24] The SOLVE/THESEO 2000 campaign had a mix of observational platforms that included satellites, ground stations, aircraft, and balloons.

\subsubsection{Satellites}

[25] Satellite observations provided a wide view of the Arctic polar vortex over the course of the 1999/2000 winter. These observations were vital to following the hemispheric evolution of ozone, PSCs, and temperatures over the course of the winter. The satellite observations provided a first look at the basic structure of these fields. These maps assisted in the flight planning, day-to-day launch schedules, and realtime analysis of observations. Five satellite instruments were principally used during the campaign: GOME, POAM, Halogen Occultation Experiment (HALOE), TOMS, and SAGE II (see Table 1 for details on the instrument investigators and observations). In addition, the UARS Microwave Limb Sounder (MLS) instrument was turned on for two short periods during the winter.

\subsubsection{POAM}

[26] The POAM III instrument was launched on board the French SPOT 4 spacecraft on 24 March 1998, into a Sunsynchronous $98.7^{\circ} \mathrm{E}$ inclination orbit. POAM III is a ninechannel visible/near-infrared photometer $(352-1020 \mathrm{~nm})$ for measuring trace constituents in the stratosphere by using solar occultation. POAM III measurements include $\mathrm{O}_{3}$, $\mathrm{H}_{2} \mathrm{O}, \mathrm{NO}_{2}$, and aerosol extinction. A detailed description of POAM and early POAM validation results are given by Lucke et al. [1999]. POAM makes 14 measurements per day in each hemisphere evenly spaced in longitude around a circle of latitude. The latitudes of POAM occultations are shown in Figure 2 as the magenta line. Because of the polar vortex's size, this sampling pattern allows POAM to obtain profiles both inside and outside the Arctic polar vortex on a daily basis throughout the winter.

\subsubsection{TOMS}

[27] The Total Ozone Mapping Spectrometer instruments form a nearly continuous record since the first was launched in late 1978 aboard the Nimbus 7 spacecraft. The most recent TOMS was launched on 2 July 1996 on the Earth Probe satellite. This instrument uses six wavelengths $(360.0,331.2$, $322.3,317.5,312.5$, and $308.6 \mathrm{~nm}$ ) in the UV portion of the spectrum to derive total column ozone amounts. The side-toside instrument scanning combined with the $39 \mathrm{~km}$ by $39 \mathrm{~km}$ nadir field of view yields complete daily coverage of total ozone across the globe. Because the instrument requires backscattered solar radiation, observations are not available 


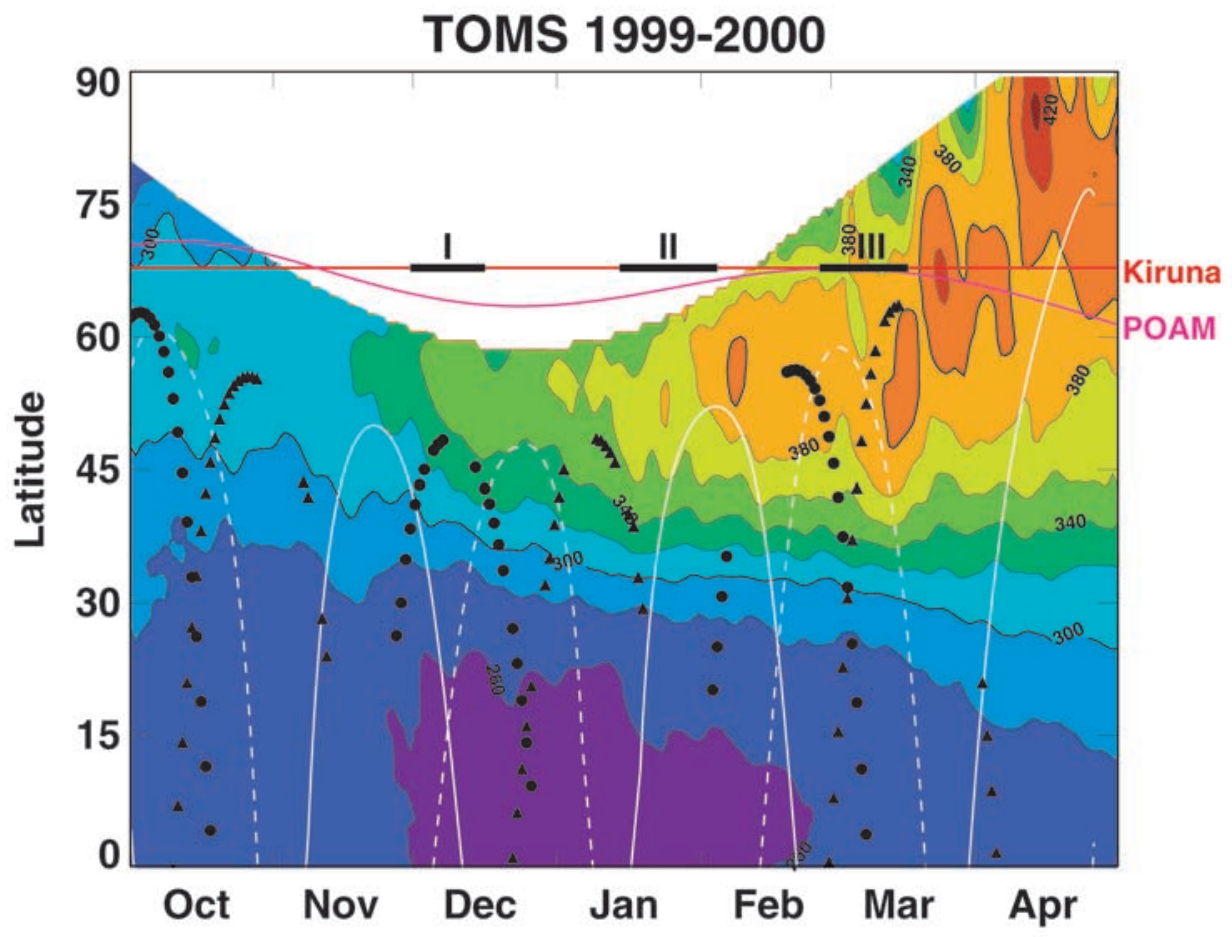

Figure 2. Zonal mean total ozone from the TOMS instrument. The latitude of Kiruna is superimposed as the red line, the occultation latitude of the POAM observations is superimposed as the magenta line, the sunrise (sunset) occultations of the HALOE instrument are superimposed as the black circles (triangles), and the sunrise (sunset) occultations of the SAGE II instrument are superimposed as the solid (dashed) white lines. Campaign phases are superimposed.

in polar night (solar zenith angles $>82^{\circ}$ ). Figure 2 displays the longitudinally averaged (zonal mean) TOMS ozone over the period of October 1999 through April 2000.

\subsubsection{GOME}

[28] The Global Ozone Monitoring Experiment was launched on 21 April 1995 on board the second European Remote Sensing Satellite (ERS 2) [Burrows et al., 1999, and references therein]. GOME is a nadir-scanning spectrometer that measures the solar radiation scattered by the atmosphere and the extraterrestrial solar irradiance in the ultraviolet and visible spectral region. The entire spectral region between 230 and $790 \mathrm{~nm}$ is observed at moderate spectral resolution of 0.2 to $0.4 \mathrm{~nm}$ in four individual linear detector arrays each having 1024 detector pixels. The field of view may be varied in size from $320 \mathrm{~km}$ by $40 \mathrm{~km}$ to $40 \mathrm{~km}$ by $40 \mathrm{~km}$ for a $1.5-\mathrm{s}$ measurement. For $\mathrm{O}_{3}$ profiling a 12-second swath is typically used, corresponding to eight 1.5-s measurements or $960 \mathrm{~km} \times 80 \mathrm{~km}$. The ERS 2 orbit is a Sun-synchronous orbit having an equator crossing time of 1030. As a result, GOME obtains full global coverage over a 3-day period at the equator. As with TOMS, GOME relies on backscattered solar radiation; hence it can only make measurements outside the region of continuous polar darkness. GOME products for the SOLVE/THESEO 2000 campaign include column $\mathrm{O}_{3}, \mathrm{BrO}, \mathrm{NO}_{2}, \mathrm{OClO}, \mathrm{H}_{2} \mathrm{CO}$, and $\mathrm{O}_{3}$ profiles.

\subsubsection{UARS HALOE}

[29] The Halogen Occultation Experiment is on the Upper Atmospheric Research Satellite (UARS) which was launched on 12 September 1991 into a $57^{\circ}$ inclined orbit. HALOE measures the absorption of solar energy due to stratospheric trace gases during spacecraft sunrise and sunset events. Vertical scans of the atmosphere are obtained by tracking the Sun position during 15 sunrise and 15 sunset occultations per day. The instantaneous vertical field of view is $1.6 \mathrm{~km}$. Daily retrievals are at approximately equal latitudes with a $24^{\circ}$ longitudinal resolution. Latitudinal sweeps occur approximately once every 30 days. The HALOE altitude range is $15 \mathrm{~km}$ to $60-130 \mathrm{~km}$, depending on the species. Vertical profiles of $\mathrm{O}_{3}, \mathrm{H}_{2} \mathrm{O}, \mathrm{NO}_{2}$, temperature, and pressure are obtained by using broadband techniques. Vertical profiles of $\mathrm{HCl}, \mathrm{HF}, \mathrm{CH}_{4}$, and $\mathrm{NO}$ rely on gas filter radiometer techniques [Russell et al., 1993]. Aerosol extinction coefficient profiles and sulfate aerosol surface area densities (SAD) are inferred [Hervig et al., 1996].

[30] Version 19 (third public release) of the HALOE retrieval was used during SOLVE/THESEO 2000. HALOE made extensive observations of the early vortex formation during October and November 1999 with 18\% of Northern Hemisphere observations occurring within the Arctic protovortex. Figure 2 displays the HALOE occultations for both sunrise (black circles) and sunset (black triangles). From mid-February to mid-March, HALOE occultations returned to high northern latitudes, and once again the vortex was sampled.

\subsubsection{SAGE II}

[31] The Stratospheric Aerosol and Gas Experiment II sensor was launched into a $57^{\circ}$ inclination orbit aboard the Earth Radiation Budget Satellite (ERBS) in October 1984. During each sunrise and sunset encountered by the orbiting spacecraft, the instrument uses the solar occultation techni- 
que to measure attenuated solar radiation through the Earth's limb in seven channels centered at wavelengths ranging from 0.385 to $1.02 \mu \mathrm{m}$. The exo-atmospheric solar irradiance is also measured in each channel during each event for use as a reference in determining limb transmittances.

[32] The transmittance measurements are inverted by using the "onion-peeling" approach to yield 1-km vertical resolution profiles of aerosol extinction (at 0.385, 0.453, 0.525 , and $1.02 \mu \mathrm{m}), \mathrm{O}_{3}, \mathrm{NO}_{2}$, and $\mathrm{H}_{2} \mathrm{O}$. Although the focus of the measurements is on the lower and middle stratosphere, retrieved profiles of aerosol, $\mathrm{H}_{2} \mathrm{O}$, and $\mathrm{O}_{3}$ often extend well into the troposphere under nonvolcanic and cloud-free conditions.

[33] The sampling coverage for SAGE II varies in latitude from approximately $60^{\circ} \mathrm{N}$ to $80^{\circ} \mathrm{S}$ during the winter, as shown by the solid and dashed white lines in Figure 2. A detailed description of SAGE II can be found in the work of McCormick et al. [1979] and Cunnold et al. [1991].

\subsubsection{UARS MLS}

[34] The Microwave Limb Sounder on UARS observes microwave emission from Earth's limb to measure vertical profiles of selected chemical species and temperature. Waters [1993] describes the measurement technique, and Waters et al. [1999] summarize results obtained through 1999. The instrument [Barath et al., 1993] operates at 63, 183 , and $205 \mathrm{GHz}$. The instrument's calibration is described by Jarnot et al. [1996].

[35] MLS was in standby mode for most of 1999-2000. It was operated during two periods, $2-13$ February and $27-$ 29 March 2000 to measure Arctic $\mathrm{ClO}, \mathrm{HNO}_{3}$, and $\mathrm{O}_{3}$ with the 205-GHz radiometer [Santee et al., 2000]. Horizontal coverage was limited during these two observing periods, and the vertical resolution of the profiles was estimated to be $6 \mathrm{~km}, 4 \mathrm{~km}$, and $4 \mathrm{~km}$ for $\mathrm{HNO}_{3}, \mathrm{ClO}$, and $\mathrm{O}_{3}$, respectively [Santee et al., 2000].

\subsubsection{Ground Stations}

[36] A total of 20 ground stations with 51 instruments were involved in the SOLVE/THESEO 2000 campaign. Lidars, Système D'Analyse par Observations Zenithales (SAOZs), Dobsons, differential optical absorption spectrometers (DOAS), microwave radiometers, Fourier transform infrareds (FTIR), ozonesondes, and backscatter-sondes provided continuous profiles of $\mathrm{O}_{3}, \mathrm{ClO}, \mathrm{N}_{2} \mathrm{O}, \mathrm{CH}_{4}, \mathrm{HNO}_{3}, \mathrm{NO}, \mathrm{HCl}$, $\mathrm{HF}$, aerosol, and wind as well as columnar densities of $\mathrm{O}_{3}$, $\mathrm{ClO}, \mathrm{N}_{2} \mathrm{O}, \mathrm{CH}_{4}, \mathrm{HNO}_{3}, \mathrm{NO}, \mathrm{HCl}, \mathrm{HF}, \mathrm{NO}_{2}, \mathrm{BrO}, \mathrm{CO}$, $\mathrm{ClONO}_{2}, \mathrm{COF}_{2}$, and $\mathrm{OClO}$. Most lidars also measured temperature at high altitude $(<10 \mathrm{hPa})$. Table 2 lists the sites and observations.

\subsubsection{Aircraft}

[37] Six aircraft were employed during the SOLVE/THESEO 2000 campaign. The SOLVE campaign provided the NASA ER-2 and the NASA DC-8, while the THESEO 2000 campaign provided the French ARAT, the DLR Falcon, the Swiss Lear jet, and the French Mystère 20 aircraft.

\subsubsection{ER-2}

[38] The NASA ER-2 is the civilian version of the second generation Lockheed U-2 high-altitude aircraft. NASA has employed the ER-2 on numerous field campaigns. It is capable of flights above $20 \mathrm{~km}$ (pressures less than 50 $\mathrm{hPa}$ ) for durations up to 8 hours. The ER-2 flew 13 times during the second and third phases of the campaign, including transits into and from Kiruna. The aircraft is shown in
Figure 3, with the locations of the 17 instruments. The Arena Arctica hangar at Kiruna Airport can be seen in the background of Figure 3. The ER-2 instruments are listed in Table 3 along with the principal investigators (PI), home institutions, and measurements.

\subsubsection{DC-8}

[39] The NASA DC-8 was a commercial airliner that has been converted into a flying laboratory. Like the ER-2, the DC-8 has been deployed on numerous field campaigns for investigating stratospheric ozone. The aircraft is capable of flights to an altitude of $12 \mathrm{~km}$ for durations over 10 hours. It flew 23 times during the three campaign phases. The aircraft is shown in Figure 4, with the locations of the 17 instruments. The DC- 8 instruments are listed in Table 4 along with the PI, home institutions, and measurements.

\subsubsection{ARAT}

[40] The ARAT is a turbo-prop Fokker 27-MK 700 aircraft that carries the LEANDRE backscatter lidar (PI, C. David of CNRS-SA/IPSL). It is capable of flight to an altitude of approximately $5 \mathrm{~km}$ and durations of 4 hours. The ARAT aircraft is a research facility jointly operated by the Institut National des Sciences de l'Univers (INSU-CNRS), the French Space Agency (CNES), the French National Weather Center (Météo-France), and the National Geographic Institute (IGN). LEANDRE is a backscatter lidar emitting 10 pulses s $^{-1}$ at a wavelength of $532 \mathrm{~nm}$. The backscattered signal is split into components polarized parallel and perpendicular to the laser emission. The ARAT flew on 25 January 2000 in coordination with the second PSC-Analysis balloon. Two flights were performed. The first flight was upstream toward Andøya, before the PSC-Analysis gondola launch, while the second flight was directed southeastward between Kiruna and Rovaniemi. During this second flight, the position of the balloon was communicated in real time to the ARAT through the VHF station in Esrange, allowing the aircraft to track the balloon efficiently. PSCs were observed between about 20 and $25 \mathrm{~km}$ in altitude. The ARAT also flew on 26 and 27 January 2000.

\subsubsection{Falcon}

[41] The DLR Falcon 20-E5 is a modified twin-jet-engined business aircraft capable of flights to $13.7 \mathrm{~km}$ with a maximum endurance of up to 5 hours. The Falcon carried the OLEX lidar during the SOLVE/THESEO 2000 campaign. The Ozone Lidar Experiment (OLEX) (PI, H. Flentje, DLR) measures ozone as well as depolarization- and wavelength-resolved backscatter of aerosol and PSCs. During the second phase of SOLVE/THESEO 2000 there were eight science flights (including two transit flights) of the Falcon lidar. Three of them were mountain wave flights; the other three probed the large nitric acid trihydrate (NAT) PSCs south of Spitzbergen. On 27 January the Falcon precisely tracked two balloons (HALOZ and Triple), which carried various in situ aerosol probes. Figure 5 displays the DLR Falcon parked on the ramp outside of the Arena Arctica on 31 January 2000. 2.2.3.5. Lear Jet

[42] The Swiss Air Force Lear jet carried a microwave radiometer on flights between Kiruna and Iceland, Kiruna and the North Pole, and Kiruna and the North Atlantic in the period of 8 to 14 March 2000 (PI, N. Kämpfer, University of Bern). This instrument obtained spectra of stratospheric water vapor at $183 \mathrm{GHz}$ as well as ozone at $176 \mathrm{GHz}$, from which profiles were derived over an altitude range of roughly 
Table 2. Ground Instruments During the SOLVE/THESEO 2000 Campaign

\begin{tabular}{|c|c|c|c|c|c|}
\hline Site & Latitude ${ }^{\circ} \mathrm{N}$ & Longitude ${ }^{\circ} \mathrm{E}$ & Instruments & Profile & Column \\
\hline $\begin{array}{l}\text { Observatoire } \\
\text { Haute Provence, } \\
\text { France }\end{array}$ & 43.94 & 5.71 & $\begin{array}{l}\text { lidar, } \mathrm{SAOZ} \text {, } \\
\text { Dobson, } \mathrm{O}_{3} \text { sonde, } \\
\text { DOAS }\end{array}$ & $\mathrm{O}_{3}$, aerosol & $\mathrm{O}_{3}, \mathrm{NO}_{2}, \mathrm{BrO}$ \\
\hline $\begin{array}{l}\text { Plateau de } \\
\text { Bure, France }\end{array}$ & 44.63 & 5.90 & microwave & $\mathrm{ClO}, \mathrm{O}_{3}$ & \\
\hline $\begin{array}{l}\text { Observatoire } \\
\text { de Bordeaux, } \\
\text { France }\end{array}$ & 44.83 & -0.52 & $\begin{array}{l}\text { SAOZ, Dobson, } \\
\text { microwave, DOAS }\end{array}$ & $\mathrm{O}_{3}$ & $\mathrm{O}_{3}, \mathrm{NO}_{2}, \mathrm{BrO}$ \\
\hline $\begin{array}{l}\text { Jungfraujoch, } \\
\text { Switzerland }\end{array}$ & 46.50 & 8.00 & $\begin{array}{l}\text { SAOZ, microwave, } \\
\text { FTIR }\end{array}$ & $\mathrm{ClO}$ & $\begin{array}{l}\mathrm{O}_{3}, \mathrm{NO}_{2}, \mathrm{CO}, \mathrm{CH}_{4} \\
\mathrm{HNO}_{3}, \mathrm{~N}_{2} \mathrm{O}, \mathrm{ClONO}_{2}, \\
\mathrm{HCl}, \mathrm{HF}\end{array}$ \\
\hline $\begin{array}{l}\text { Bern, } \\
\text { Switzerland }\end{array}$ & 46.95 & 7.45 & microwave & $\mathrm{O}_{3}$ & \\
\hline $\begin{array}{l}\text { Zugspitze, } \\
\text { Germany }\end{array}$ & 47.42 & 10.98 & FTIR & & $\begin{array}{l}\mathrm{O}_{3}, \mathrm{NO}_{2}, \mathrm{CO}, \mathrm{CH}_{4}, \\
\mathrm{HNO}_{3}, \mathrm{~N}_{2} \mathrm{O}, \mathrm{ClONO}_{2}, \\
\quad \mathrm{HCl}, \mathrm{HF}\end{array}$ \\
\hline $\begin{array}{l}\text { Garmisch, } \\
\text { Germany }\end{array}$ & 47.48 & 11.06 & lidar & aerosol & \\
\hline Cambridge, UK & 52.00 & 0.00 & DOAS & & $\mathrm{BrO}$ \\
\hline $\begin{array}{l}\text { Bremen, } \\
\text { Germany }\end{array}$ & 53.00 & 9.00 & DOAS & & $\mathrm{O}_{3}, \mathrm{NO}_{2}, \mathrm{BrO}$ \\
\hline Lerwick, UK & 60.13 & 1.18 & Dobson, $\mathrm{O}_{3}$ sonde & $\mathrm{O}_{3}$ & $\mathrm{O}_{3}$ \\
\hline $\begin{array}{l}\text { Harestua, } \\
\text { Norway }\end{array}$ & 60.20 & 10.80 & $\begin{array}{l}\text { DOAS, FTIR, } \\
\text { Dobson }\end{array}$ & $\mathrm{O}_{3}$ & $\begin{array}{l}\mathrm{O}_{3}, \mathrm{NO}_{2}, \mathrm{COF}_{2}, \mathrm{HNO}_{3}, \\
\mathrm{~N}_{2} \mathrm{O}, \mathrm{OClO}_{\mathrm{BrO}}, \\
\quad \mathrm{ClONO}_{2}, \mathrm{ClO}, \mathrm{HCl}, \mathrm{HF}\end{array}$ \\
\hline $\begin{array}{l}\text { Salekhard, } \\
\text { Russia }\end{array}$ & 66.70 & 66.70 & $\mathrm{SAOZ}$ & & $\mathrm{O}_{3}, \mathrm{NO}_{2}$ \\
\hline $\begin{array}{c}\text { Zhigansk, } \\
\text { Russia }\end{array}$ & 67.20 & 123.40 & $\mathrm{SAOZ}$ & & $\mathrm{O}_{3}, \mathrm{NO}_{2}$ \\
\hline $\begin{array}{l}\text { Sodankyla, } \\
\text { Finland }\end{array}$ & 67.37 & 26.65 & $\begin{array}{l}\text { back. sonde, } \mathrm{O}_{3} \\
\text { sonde, } \mathrm{SAOZ} \text {, } \\
\text { Brewer }\end{array}$ & $\mathrm{O}_{3}$, aerosol & $\mathrm{O}_{3}, \mathrm{NO}_{2}$ \\
\hline Kiruna, Sweden & 67.84 & 20.41 & DOAS, FTIR, MW & $\begin{array}{l}\mathrm{O}_{3}, \mathrm{~N}_{2} \mathrm{O}, \\
\mathrm{CH}_{4}, \mathrm{HNO}_{3}, \\
\mathrm{NO}, \mathrm{HCl}, \\
\mathrm{HF}, \mathrm{ClO}\end{array}$ & $\begin{array}{c}\mathrm{O}_{3}, \mathrm{~N}_{2} \mathrm{O}, \mathrm{CH}_{4}, \mathrm{HNO}_{3}, \\
\mathrm{NO}_{2}, \mathrm{NO}, \mathrm{ClONO}_{2}, \\
\mathrm{HCl} \mathrm{ClO}, \mathrm{COF}_{2} \\
\mathrm{HF}, \mathrm{OClO}, \mathrm{BrO}\end{array}$ \\
\hline ESRANGE, Sweden & 67.88 & 21.06 & $\begin{array}{l}\text { DOAS, lidar, } \mathrm{O}_{3} \\
\text { sonde, radar }\end{array}$ & $\begin{array}{l}\text { aerosol, } \mathrm{O}_{3}, \\
\text { wind }\end{array}$ & $\mathrm{O}_{3}, \mathrm{NO}_{2}$ \\
\hline Andøya, Norway & 69.30 & 16.00 & $\begin{array}{l}\text { lidar, } \mathrm{O}_{3} \text { sonde, } \\
\text { DOAS }\end{array}$ & $\mathrm{O}_{3}$, aerosol & $\begin{array}{l}\mathrm{O}_{3}, \mathrm{NO}_{2}, \mathrm{OClO} \\
\quad \mathrm{BrO}\end{array}$ \\
\hline Scoresbysund, Greenland & 70.48 & -21.97 & $\mathrm{SAOZ}$ & & $\mathrm{O}_{3}, \mathrm{NO}_{2}$ \\
\hline Thule, Greenland & 76.53 & -68.74 & $\begin{array}{l}\mathrm{O}_{3} \text { sonde, DOAS, } \\
\text { SAOZ, back. sonde }\end{array}$ & $\mathrm{O}_{3}$, aerosol & $\mathrm{O}_{3}, \mathrm{NO}_{2}, \mathrm{OClO}$ \\
\hline Ny Alesund, Spitsbergen & 78.92 & 11.93 & $\begin{array}{l}\text { FTIR, SAOZ, lidar, } \\
\text { DOAS, microwave, } \\
\mathrm{O}_{3} \text { sonde, back. } \\
\text { sonde }\end{array}$ & $\begin{array}{l}\mathrm{O}_{3} \text {, aerosol, } \\
\quad \mathrm{ClO}\end{array}$ & $\begin{array}{c}\mathrm{O}_{3}, \mathrm{NO}_{2}, \mathrm{OClO}, \mathrm{BrO}, \\
\mathrm{CO}, \mathrm{CH}_{4}, \mathrm{HNO}_{3}, \\
\mathrm{~N}_{2} \mathrm{O}, \mathrm{ClO}, \mathrm{HCl}, \mathrm{HF}\end{array}$ \\
\hline
\end{tabular}

$20-70 \mathrm{~km}$. After the Arctic flights, the plane flew south to the Canary Islands to extend the latitude range of the measurements to $29^{\circ} \mathrm{N}$.

\subsubsection{Mystère 20}

[43] The French Mystère 20 is a twin-turbofan-engine aircraft built by Dassault. The Mystère is capable of 3-hour flights to a maximum altitude of approximately $13 \mathrm{~km}$ and a range of approximately $1800 \mathrm{~km}$. During SOLVE/THESEO 2000 , it carried the Alto ozone lidar (PIs, S. Godin and A. Hauchecorne, CNRS-SA/IPSL), which is a differential absorption laser (DIAL) system capable of measuring ozone profiles from $1 \mathrm{~km}$ above the plane to about $20 \mathrm{~km}$. The objective for the Mystère was to measure polar ozone filaments that were breaking off of the polar vortex. The Mystère was flown on 28 February, 7 March, 26 March, and 28 March 2000 and was operated from Creil Airport near Paris.

\subsubsection{Balloons}

\subsubsection{OMS in Situ}

[44] The NASA OMS in situ payload was launched on 19 November 1999 and 5 March 2000, with both flights inside the Arctic vortex. In situ measurements of the vertical profiles of a range of atmospheric tracers were made (see Table 5). The Observations of the Middle Stratosphere (OMS) in situ gondola included the first four instruments of the table, plus the frost point hygrometer as a free flying package in parallel with the OMS in situ payload.

\subsubsection{OMS Remote}

[45] The OMS remote payload consisted of two instruments (see Table 5). The Jet Propulsion Laboratory (JPL) MkIV infra-red interferometer made profile measurements of a wide range of species in solar absorption mode by observing sunrise or sunset from a balloon. The Submillimeterwave Limb Sounder (SLS) is a heterodyne radiometer measuring thermal emission spectra. This payload made two flights, both inside the Arctic vortex. The first was early in the winter on 3 December 1999, and the second was on 15 March 2000 just prior to a major split in the vortex.

\subsubsection{MIR}

[46] The Montgolfière Infrarouge (MIR) balloons are capable of long-duration flights and have been used in the 


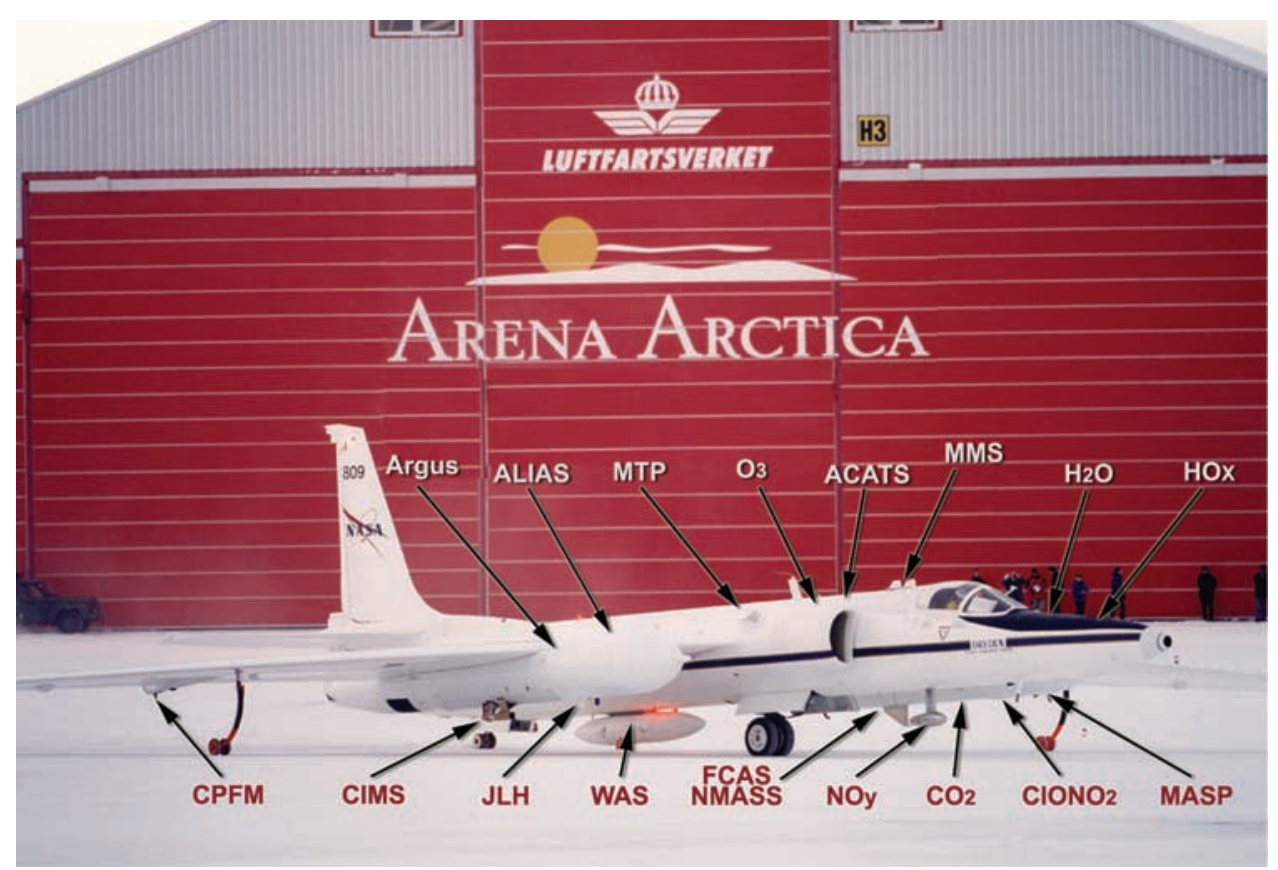

Figure 3. NASA ER-2 with payload layout in front of the Arena Arctica in Kiruna, Sweden.

Arctic vortex and in the tropics. The objective of the SOLVE/ THESEO 2000 flights was to complement the measurements of 1999 by two new instrumented scientific MIR flights in the vortex as well as an additional technical MIR, all carrying payloads recovered in 1999. The first two MIRs were launched on the same day (18 February 2000) when the vortex passed rapidly above Kiruna. They lasted for 2 and 18 days, both being automatically cut down when reaching $55^{\circ} \mathrm{N}$. Their respective payloads were recovered near Hudson Bay and in Belarus near Minsk. The third (technical) balloon failed during the initial ascent, and no useful measurements were made. The data include temperature, pressure, altitude,

Table 3. ER-2 Payload

\begin{tabular}{|c|c|c|c|}
\hline Instrument & Investigator & Institution & Measurements \\
\hline ACATS & J. W. Elkins & NOAA/CMDL & $\begin{array}{l}\text { CFC-11, CFC-12, CFC-113, } \\
\mathrm{CH}_{3} \mathrm{CCl}_{3}, \mathrm{CCl}_{4}, \text { halon-1211, } \\
\mathrm{CHCl}_{3}, \mathrm{CH}_{4}, \mathrm{H}_{2}, \mathrm{~N}_{2} \mathrm{O}, \mathrm{SF}_{6}\end{array}$ \\
\hline ALIAS & C. Webster & JPL & $\mathrm{HCl}, \mathrm{CO}, \mathrm{CH}_{4}, \mathrm{~N}_{2} \mathrm{O}$ \\
\hline Argus & H. Jost & NASA/ARC & $\mathrm{N}_{2} \mathrm{O}, \mathrm{CH}_{4}$ \\
\hline CIMS & $\begin{array}{l}\text { P. Wennberg } \\
\text { F. Eisele }\end{array}$ & $\begin{array}{l}\text { Cal. Tech. } \\
\text { NCAR }\end{array}$ & $\mathrm{HNO}_{3}$ \\
\hline $\mathrm{ClONO}_{2}$ & R. Stimpfle & Harvard Univ. & $\mathrm{ClO}, \mathrm{ClONO}_{2}, \mathrm{Cl}_{2} \mathrm{O}_{2}, \mathrm{BrO}$ \\
\hline $\mathrm{CO}_{2}$ & S. Wofsy & Harvard Univ. & $\mathrm{CO}_{2}$ \\
\hline CPFM & C. T. McElroy & AES-Canada & $\begin{array}{l}\text { UV-vis. spectra, column } \mathrm{O}_{3} \\
\quad J \text { values, albedo }\end{array}$ \\
\hline $\begin{array}{l}\text { FCAS } \\
\text { N-MASS } \\
\text { Impactor }\end{array}$ & J. Wilson & Univ. of Denver & $\begin{array}{l}0.07-\text { to } 1-\mu \mathrm{m} \text { particles } \\
\text { 4- to } 60-\mathrm{nm} \text { particles } \\
\text { particle composition } \\
\text { and structure }\end{array}$ \\
\hline $\mathrm{H}_{2} \mathrm{O}$ & $\begin{array}{l}\text { E. Weinstock } \\
\text { R. Spackman }\end{array}$ & Harvard Univ. & $\mathrm{H}_{2} \mathrm{O}$ \\
\hline $\mathrm{HO}_{\mathrm{x}}$ & $\begin{array}{l}\text { T. Hanisco, } \\
\text { J. Smith }\end{array}$ & Harvard Univ. & $\mathrm{OH}, \mathrm{HO}_{2}$ \\
\hline JLH & R. Herman & JPL & $\mathrm{H}_{2} \mathrm{O}$ \\
\hline MASP & $\begin{array}{l}\text { D. Baumgardner } \\
\text { B. Gandrud }\end{array}$ & NCAR & $0.4-$ to $20-\mu \mathrm{m}$ particles \\
\hline MMS & T. P. Bui & NASA/ARC & $\mathrm{T}, \mathrm{P}, \mathrm{U}, \mathrm{V}, \mathrm{W}$, position \\
\hline MTP & M. Mahoney & JPL & temp. profiles \\
\hline $\mathrm{NO}_{2}$ & $\begin{array}{l}\text { K. Perkins } \\
\text { E. Lanzendorf }\end{array}$ & Harvard Univ. & $\mathrm{NO}_{2}$ \\
\hline $\begin{array}{l}\mathrm{NO}_{y} \\
\text { Ozone }\end{array}$ & $\begin{array}{l}\text { D. Fahey, R. Gao } \\
\text { E. Richards }\end{array}$ & $\begin{array}{l}\text { NOAA/AL } \\
\text { NOAA/AL }\end{array}$ & $\begin{array}{l}\mathrm{NO}, \mathrm{NO}_{\mathrm{y}} \\
\mathrm{O}_{3}\end{array}$ \\
\hline WAS & $\begin{array}{l}\text { E. Atlas } \\
\text { S. Schauffler }\end{array}$ & NCAR & $\begin{array}{l}\mathrm{CFCs} \text {, halons, } \mathrm{HCFCs}, \mathrm{N}_{2} \mathrm{O}, \\
\mathrm{CH}_{4} \text {, HFCs, PFCs, } \\
\text { hydrocarbons, etc. }\end{array}$ \\
\hline
\end{tabular}




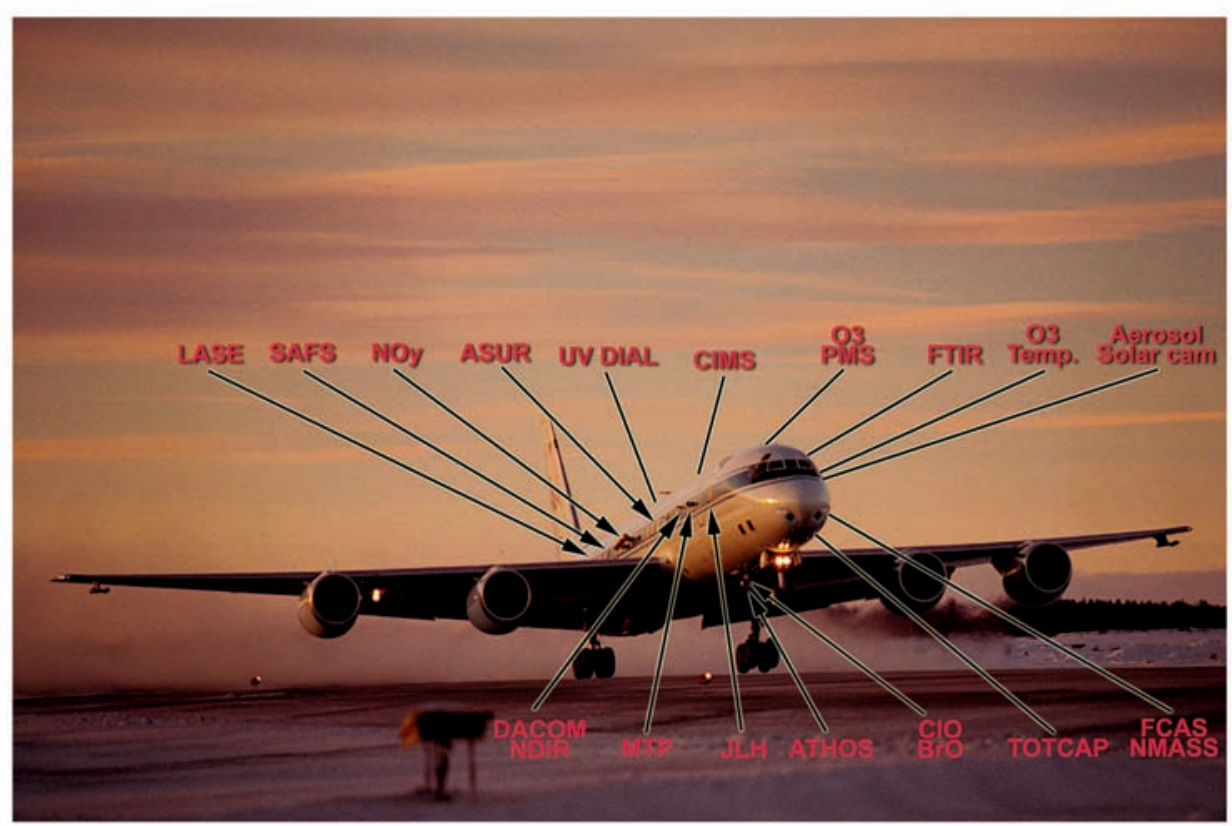

Figure 4. NASA DC-8 taking off from Kiruna with its payload layout.

and IR radiation measurements every 15 min along the two flights, five $\mathrm{CH}_{4}$ and $\mathrm{H}_{2} \mathrm{O}$ profiles, and ten $\mathrm{O}_{3}, \mathrm{NO}_{2}$ and OClO profiles.

\subsubsection{PSC-Analysis}

[47] The PSC-Analysis balloon-borne payload was launched from Esrange on 19 January and 25 January 2000 for detailed studies of polar stratospheric cloud par- ticles (see Figure 6). The payload consisted of four instruments (see Table 5). The balloon altitude was controlled by CNES, and on each flight the balloon went up and down, passing through PSC layers multiple times.

\subsubsection{HALOZ}

[48] A series of five small balloons carrying instruments to address the issue of Halogen-Catalyzed Ozone loss

Table 4. DC-8 Payload

\begin{tabular}{|c|c|c|c|}
\hline Instrument & Investigator & Institution & Measurements \\
\hline LASE & E. Browell & NASA/LRC & $\mathrm{H}_{2} \mathrm{O}$ and aerosol back. prof. \\
\hline UV DIAL & E. Browell & NASA/LRC & $\mathrm{O}_{3}$ and aerosol prof. \\
\hline SAFS & R. Shetter & NCAR & $\begin{array}{l}\text { actinic flux } 280-535 \mathrm{~nm} \\
J \text { values }\end{array}$ \\
\hline ASUR & K. Kunzi & Univ. of Bremen & $\begin{array}{l}\text { prof. of } \mathrm{ClO}, \mathrm{HCl}, \mathrm{N}_{2} \mathrm{O}, \mathrm{O}_{3} \text {, } \\
\mathrm{HNO}_{3}, \mathrm{CH}_{3} \mathrm{Cl}, \mathrm{H}_{2} \mathrm{O}, \mathrm{HO}_{2}, \mathrm{BrO}\end{array}$ \\
\hline CIMS & A. Viggiano & AF Research Lab. & $\mathrm{SO}_{2}, \mathrm{HCN}, \mathrm{HNO}_{3}$ \\
\hline PMS & B. Anderson & NASA/LRC & $\begin{array}{l}0.1-\text { to } 3-\mu \mathrm{m} \text { particles, } \\
\text { cn concentrations }\end{array}$ \\
\hline $\mathrm{O}_{3}$ & M. Avery & NASA/LRC & $\mathrm{O}_{3}$ \\
\hline Dew point & J. Barrick & NASA/LRC & dew point \\
\hline FTIR & W. Mankin & NCAR & 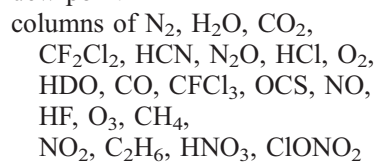 \\
\hline $\mathrm{NO}_{y}$ & Y. Kondo & Univ. of Tokyo & $\mathrm{NO}, \mathrm{NO}_{y}$ \\
\hline AEROTEL & $\begin{array}{l}\text { T. McGee, J. Burris } \\
\text { C. Hostetler }\end{array}$ & $\begin{array}{l}\text { NASA/GSFC } \\
\text { NASA/LRC }\end{array}$ & $\begin{array}{l}\mathrm{O}_{3} \text {, temp., aerosol prof. } \\
\text { prof. aerosol scattering, } \\
\text { backscatter, polarization }\end{array}$ \\
\hline Solar Cam & C. Hostetler & NASA/LRC & solar images \\
\hline DACOM & G. Sachse & NASA/LRC & $\mathrm{CO}, \mathrm{CH}_{4}, \mathrm{~N}_{2} \mathrm{O}$ \\
\hline DLH & G. Sachse, J. Podolske & LRC/Ames & $\mathrm{H}_{2} \mathrm{O}$ \\
\hline NDIR & S. Vay & NASA/LRC & $\mathrm{CO}_{2}$ \\
\hline JLH & R. Herman & JPL & $\mathrm{H}_{2} \mathrm{O}$ \\
\hline ATHOS & B. Brune & Penn. State & $\mathrm{OH}, \mathrm{HO}_{2}$ \\
\hline $\mathrm{ClO} / \mathrm{BrO}$ & D. Toohey & Univ. of Colorado & $\mathrm{ClO}$ \\
\hline TOTCAP & L. Avallone & Univ. of Colorado & $\begin{array}{l}\mathrm{O}_{3} \text {, total } \mathrm{H}_{2} \mathrm{O}, \mathrm{CO}_{2}, \mathrm{~F} 11, \mathrm{~F} 12, \\
\text { F113, halon-1211, } \mathrm{CCl}_{4}\end{array}$ \\
\hline FCAS II NMASS & J. Reeves & Univ. of Denver & $\begin{array}{l}0.08 \text { - to } 2-\mu \mathrm{m} \text { particles } \\
4 \text { - to } 100-\mathrm{nm} \text { particles }\end{array}$ \\
\hline MTP & M. Mahoney & JPL & Temp. profiles \\
\hline
\end{tabular}




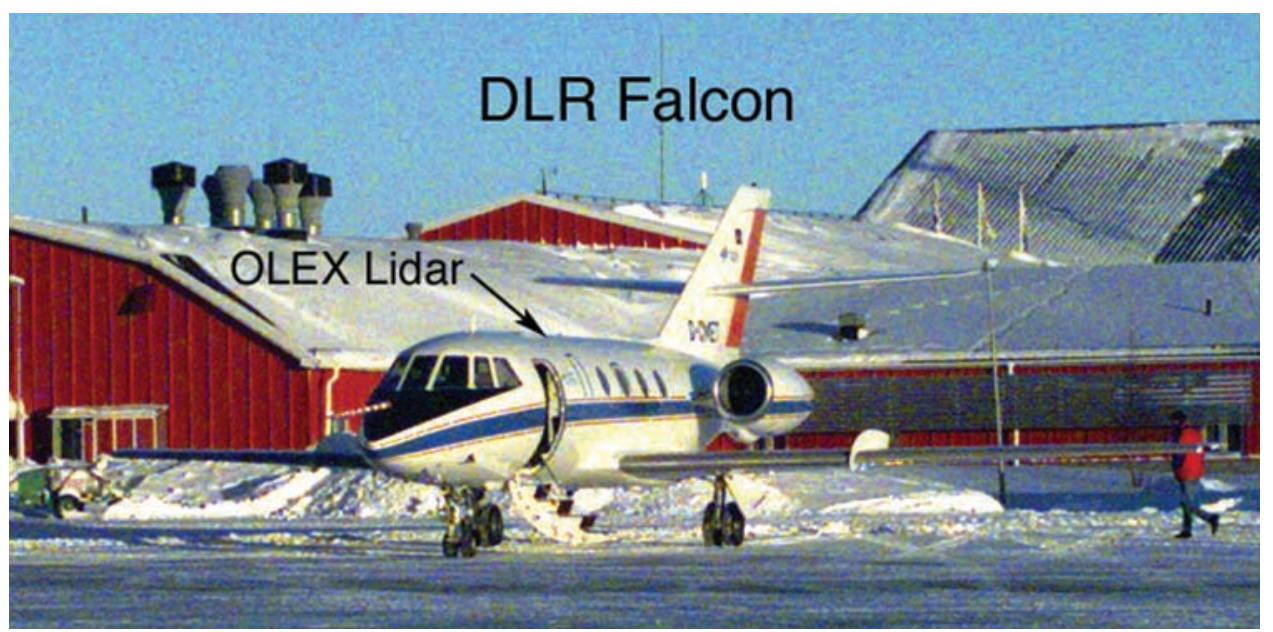

Figure 5. DLR Falcon with OLEX lidar at Kiruna Airport.

Table 5. Balloon Payloads

\begin{tabular}{|c|c|c|c|c|}
\hline Balloon & Instrument & Principal Investigator & Institution & Observations \\
\hline \multirow[t]{5}{*}{ OMS in situ } & ALIAS-II & C. Webster & JPL & $\mathrm{HCl}, \mathrm{CH}_{4}, \mathrm{~N}_{2} \mathrm{O}$ \\
\hline & ozone & J. Margitan & JPL & \\
\hline & $\mathrm{CO}^{2}$ Analyzer & S. Wofsy & Harvard Univ. & $\mathrm{CO}_{2}$ \\
\hline & LACE & J. Elkins & NOAA/CMDL & $\begin{array}{l}\text { CFC-11, CFC-12, } \\
\text { CFC-113, halon-1211, } \mathrm{CHCl}_{3} \text {, } \\
\mathrm{CH}_{3} \mathrm{CCl}_{3}, \mathrm{CCl}_{4}, \mathrm{SF}_{6} \text { and } \mathrm{N}_{2} \mathrm{O}\end{array}$ \\
\hline & $\begin{array}{l}\text { frost point } \\
\text { hygrometer }\end{array}$ & S. Olrmans & NOAA/CMDL & $\mathrm{H}_{2} \mathrm{O}$ \\
\hline \multirow[t]{2}{*}{ OMS remote } & $\begin{array}{l}\text { MkIV } \\
\text { interferometer }\end{array}$ & G. Toon & JPL & $\begin{array}{l}\mathrm{H}_{2} \mathrm{O}, \mathrm{O}_{3}, \mathrm{~N}_{2} \mathrm{O}, \mathrm{CO}, \mathrm{CH}_{4}, \mathrm{NO}, \\
\mathrm{NO}_{2}, \mathrm{HNO}_{3}, \mathrm{HNO} \mathrm{H}_{4}, \mathrm{~N}_{2} \mathrm{O}_{5}, \mathrm{H}_{2} \mathrm{O}_{2}, \\
\mathrm{ClNO}_{3}, \mathrm{HOCl}, \mathrm{HCl}, \mathrm{HF}, \mathrm{COF}_{2}, \mathrm{CF}_{4}, \\
\mathrm{SF}_{6}, \mathrm{CFC}-11, \mathrm{CFC}-12, \mathrm{CCl}_{4}, \\
\mathrm{CH}_{3} \mathrm{Cl}, \mathrm{CO}_{2}\end{array}$ \\
\hline & SLS & R. Stachnik & JPL & $\mathrm{ClO}, \mathrm{O}_{3}, \mathrm{HCl}, \mathrm{HNO}_{3}, \mathrm{~N}_{2} \mathrm{O}$ \\
\hline MIR & MIR & J. P. Pommereau & CNRS-SA & $\mathrm{T}, \mathrm{P}, \mathrm{CH}_{4}, \mathrm{H}_{2} \mathrm{O}, \mathrm{O}_{3}, \mathrm{NO}_{2}, \mathrm{OClO}$ \\
\hline \multirow[t]{4}{*}{ PSC analysis } & ACMS & J. Schreiner & MPIK & aerosol composition \\
\hline & aerosol counter & T. Deshler & Univ. of Wyoming & $\mathrm{CN}$, aerosols $>0.3 \mu \mathrm{m}$ \\
\hline & backscatter sonde & $\begin{array}{l}\text { A. Adriani, J. Rosen, } \\
\text { N. Larsen }\end{array}$ & $\begin{array}{l}\text { CNR-IFA } \\
\text { Univ. of Wyoming } \\
\text { DMI }\end{array}$ & particle concentration \\
\hline & $\begin{array}{l}\text { frost point } \\
\text { hygrometer }\end{array}$ & J. Ovarlez & LMD & $\mathrm{H}_{2} \mathrm{O}$ \\
\hline \multirow[t]{5}{*}{ HALOZ } & $\mathrm{ClO}$ and $\mathrm{BrO}$ & D. Toohey & Univ. of Colorado & $\mathrm{ClO}, \mathrm{BrO}$ \\
\hline & particle counter & T. Deshler & Univ. of Wyoming & particle size distributions \\
\hline & $\begin{array}{l}\text { DIRAC - gas } \\
\text { chromatograph }\end{array}$ & A. Robinson & Univ. of Cambridge & CFC-11 \\
\hline & DESCARTES & H. Nilsson & IRF & CFC-11 \\
\hline & & F. Danis & Univ. of Cambridge & \\
\hline \multirow[t]{5}{*}{ Triple } & $\mathrm{ClO}$ and $\mathrm{BrO}$ & F. Stroh & FZ Jülich & $\mathrm{ClO}, \mathrm{BrO}$ \\
\hline & $\begin{array}{l}\text { BONBON - } \\
\text { cryogenic WAS }\end{array}$ & $\begin{array}{l}\text { A. Engel } \\
\text { U. Schmidt }\end{array}$ & U. Frankfurt & $\mathrm{N}_{2} \mathrm{O}, \mathrm{CFCs}, \mathrm{CH}_{4}, \mathrm{HCFCs}, \mathrm{SF}_{6}$ \\
\hline & $\begin{array}{l}\text { FISH - Lyman - } \\
\text { alpha hygrometer }\end{array}$ & C. Schiller & FZ Jülich & $\mathrm{H}_{2} \mathrm{O}$ \\
\hline & filter UV radiometer & C. Schiller & FZ Jülich & actinic flux \\
\hline & aeosol size distributions & J. Ovarlez & CNRS-LMD & aerosol size distribution \\
\hline \multirow[t]{2}{*}{ LPMA/DOAS } & FTIR & C. Camy-Peyret & UPMC & $\begin{array}{l}\mathrm{HCl}, \mathrm{ClONO}_{2}, \mathrm{CH}_{4}, \mathrm{~N}_{2} \mathrm{O}, \mathrm{HNO}_{3}, \\
\mathrm{NO}_{2}, \mathrm{NO}, \mathrm{O}_{3}, \mathrm{HF}\end{array}$ \\
\hline & DOAS & K. Pfeilsticker & Univ. of Heidelberg & $\begin{array}{l}\mathrm{O}_{3}, \mathrm{BrO}, \mathrm{IO}, \mathrm{NO}_{2}, \mathrm{O}_{4}, \mathrm{OClO} \\
\quad \text { and OIO }\end{array}$ \\
\hline SALOMON & UV-vis spectrometer & M. Pirre & LPCE & $\begin{array}{l}\mathrm{O}_{3}, \mathrm{NO}_{2}, \mathrm{NO}_{3}, \mathrm{OClO} \text {, aerosol } \\
\quad \text { extinction }\end{array}$ \\
\hline \multirow{6}{*}{$\begin{array}{l}\text { Micro Radibal } \\
\text { SAOZ }\end{array}$} & radiance and polarization & C. Brogniez & Univ. of Lille & radiance and polarization \\
\hline & SAOZ & J.-P. Pommereau & CNRS-SA & $\mathrm{O}_{3}, \mathrm{NO}_{2}, \mathrm{OClO}, \mathrm{BrO}$ \\
\hline & DESCARTES & H. Nilsson & IRF & CFC-11 \\
\hline & & F. Danis & Univ. of Cambridge & \\
\hline & $\begin{array}{l}\text { DIRAC - gas } \\
\text { chromatograph }\end{array}$ & A. Robinson & Univ. of Cambridge & CFC-11 \\
\hline & Methane TDL & R. Jones & Univ. of Cambridge & $\mathrm{CH}_{4}$ \\
\hline
\end{tabular}




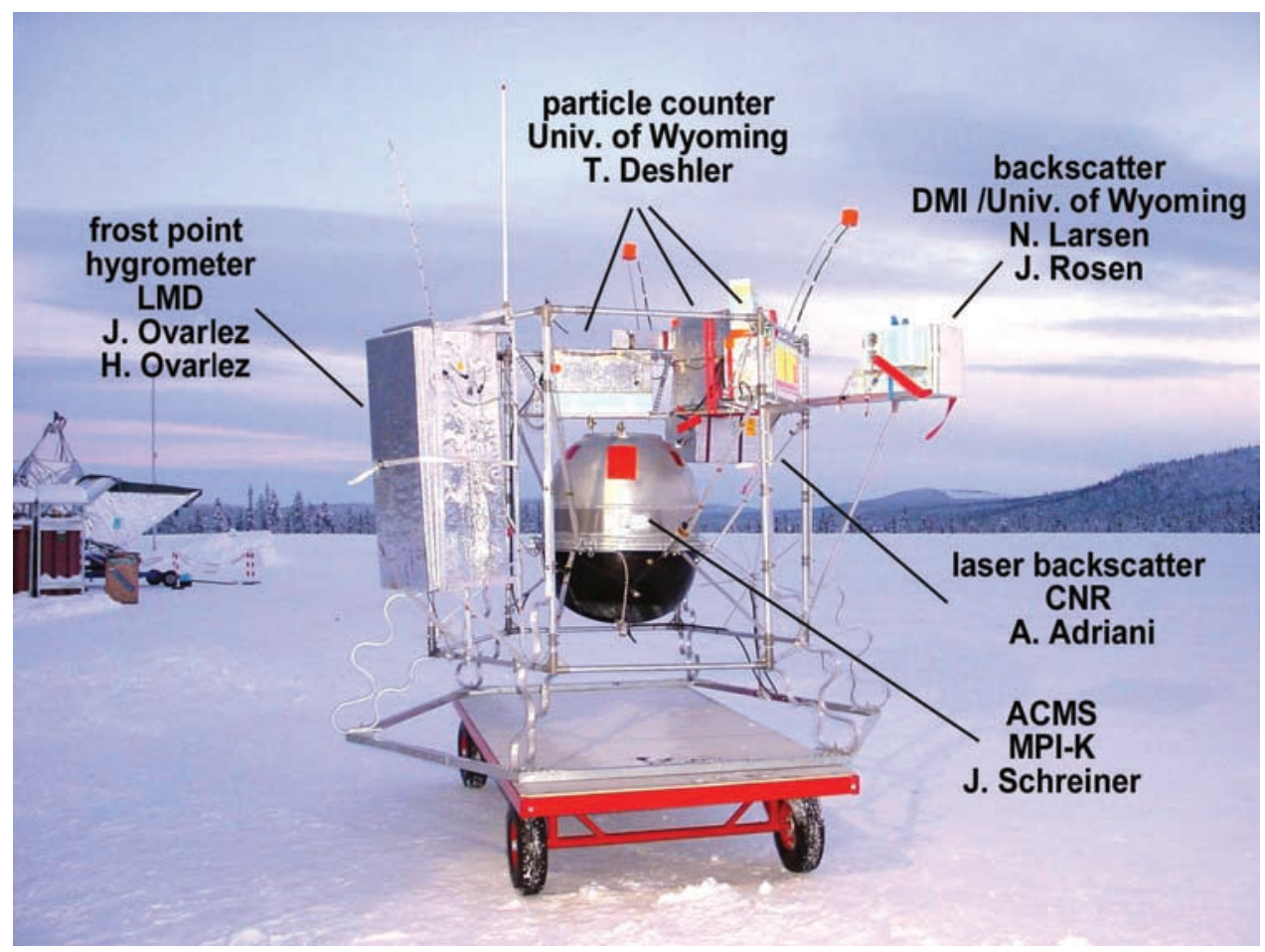

Figure 6. PSC-Analysis balloon gondola on the main launch pad at Esrange.

(HALOZ) were launched from Kiruna, Sweden, between January and April 2000. A critical subset of species involved in ozone loss were measured in situ with lightweight instruments. $\mathrm{ClO}$ and $\mathrm{BrO}$ were measured by using a resonance fluorescence instrument, particle number and size distributions were measured by optical scattering, and CFC-11 was measured by the DIRAC in situ gas chromatograph. Ozone was measured by an ozonesonde. Two distinct payload configurations were used for maximum launch flexibility. The first, which consisted of all the instruments, was launched twice (19 January and 27 January 2000) when PSCs were observed visually. For the last three flights (1 March, 8 March, and 4 April), the particle instruments were removed. On 1 March and 4 April, CFC-11 was measured by the DESCARTES grab sampler rather than DIRAC. All flights took place inside the vortex.

\subsubsection{Triple}

[49] The Triple payload included five instruments (see Table 5). This payload was flown on 27 January 2000 and 1 March 2000 from Esrange, separated by a few hours from two of the HALOZ flights. Both flights were made inside the polar vortex.

\subsubsection{LPMA/DOAS}

[50] The Limb Profile Monitor of the Atmosphere (LPMA)/DOAS payload was launched from Esrange on 18 February 2000. Measurements were made by FTIR and DOAS instruments (see Table 5). These spectrometers simultaneously measured vertical profiles of $\mathrm{HCl}, \mathrm{ClONO}_{2}$, $\mathrm{CH}_{4}, \mathrm{~N}_{2} \mathrm{O}, \mathrm{HNO}_{3}, \mathrm{NO}_{2}, \mathrm{NO}, \mathrm{O}_{3}$, and $\mathrm{HF}$ in the infrared spectral range and $\mathrm{O}_{3}, \mathrm{BrO}, \mathrm{IO}, \mathrm{NO}_{2}, \mathrm{O}_{4}, \mathrm{OClO}$, and $\mathrm{OIO}$ in the UV-visible spectral range.

\subsubsection{SALOMON}

[51] SALOMON is a UV-visible spectrometer $(350-700$ $\mathrm{nm}$ ) that uses the Moon as the light source (see Table 5). Two SALOMON flights were performed in different geophysical conditions, allowing a comparison of various aspects of the stratospheric polar chemistry to be made. The first flight was on 23 January 2000 at the edge of the vortex. The flight was stopped early for safety reasons, and observations were made in the range $13-20 \mathrm{~km}$. The second flight occurred on 22 February 2000. The measurements were made in warm conditions outside the polar vortex. The full Moon rise was observed, giving profiles between 16 and $30 \mathrm{~km}$.

\subsubsection{Micro-Radibal}

[52] Micro-Radibal is a balloon-borne instrument developed to study stratospheric aerosols; it measures the radiance and polarization of scattered sunlight at 730,865 , 1000 , and $1620 \mathrm{~nm}$. The gondola rotates around a vertical axis, and observations are made under various scattering angles during the ascent of the balloon. Micro-Radibal flew on 26 January 2000 from Esrange and made measurements between 13 and $23 \mathrm{~km}$.

\subsubsection{SAOZ Balloon Flights}

[53] The SAOZ is a UV-visible spectrometer that measures in the wavelength region 300-600 nm. During SOLVE/THESEO 2000, six flights were made inside the vortex and 2 flights were outside (see Figure 7). The basic SAOZ provides $\mathrm{O}_{3}, \mathrm{NO}_{2}$, and $\mathrm{OClO}$ vertical profiles. A UV-enhanced version, SAOZ-BrO, provided $\mathrm{BrO}$ and $\mathrm{OClO}$ vertical profiles on three flights inside the vortex and on all flights outside the vortex. One flight inside the vortex was made in parallel with a HALOZ flight to allow a comparison with the $\mathrm{ClO} / \mathrm{BrO}$ resonance-fluorescence instrument. In addition, tracer profiles were measured on all flights with either the DESCARTES or DIRAC instruments or the methane tunable diode laser (TDL) instrument. In total, DESCARTES made 11 flights during this winter, 10 in conjunction with other instruments (OMS remote, HALOZ, OMS in situ, SAOZ) and one solo flight in early winter (11 November 1999). DIRAC made four flights in 


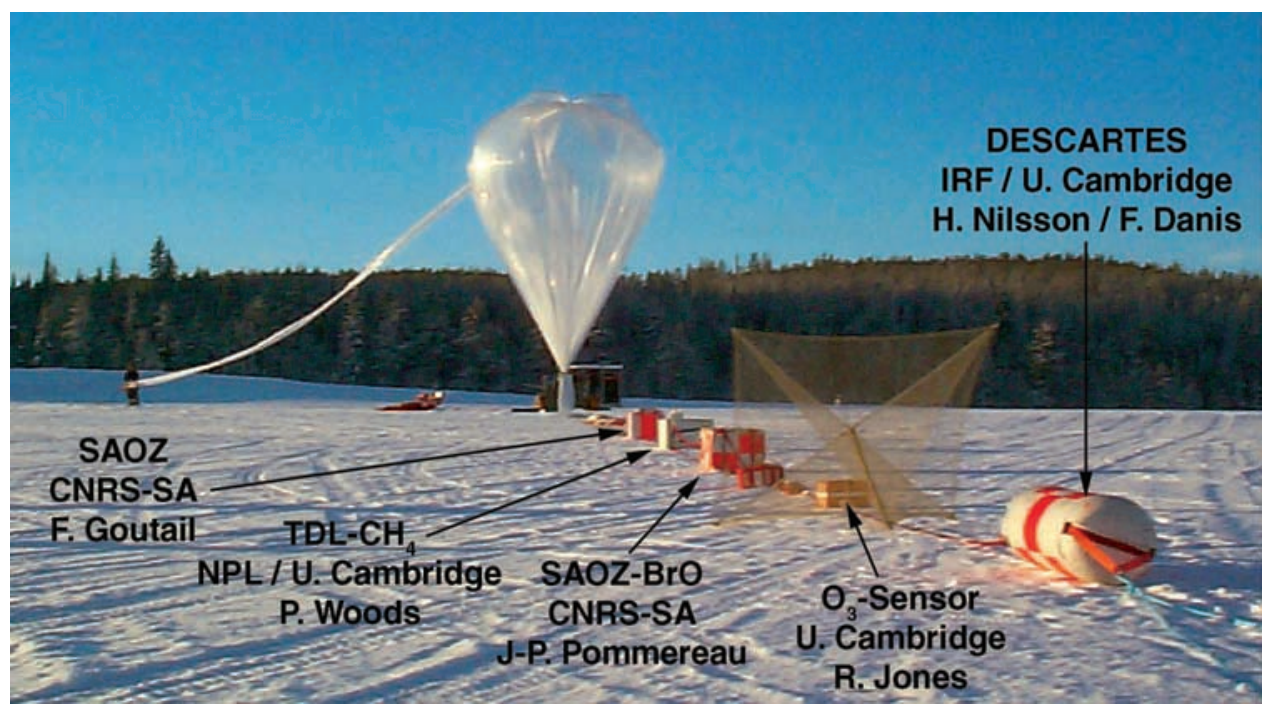

Figure 7. Preparation for launch of the small balloon payload at Esrange on 13 February 2000.

conjunction with other instruments (three with HALOZ and one with OMS in situ). These flights were made to facilitate comparisons with the OMS in situ measurements.

\subsubsection{Ozonesonde Stations}

[54] During SOLVE/THESEO 2000, over 650 ozonesondes were launched from a network of 36 stations in 20 countries at middle and high latitudes in the Northern Hemisphere (see Figure 1). Many of these stations were underneath the Arctic vortex at various times, and so a high proportion of the ozonesondes made measurements inside the Arctic vortex. The ozonesondes were either part of regular programs or were launched in response to requests within the coordinated Match program or in conjunction with aircraft or balloon flights. Figure 8 displays the mixing ratios from the ozonesondes launched from the NDSC NyÅlesund site on Spitsbergen Island.

\subsection{Flights and Launches}

[55] The aircraft and balloon flights made during the SOLVE/THESEO 2000 campaign are shown in Tables 6a, $6 \mathrm{~b}$, and $6 \mathrm{c}$, divided according to the three intensive measurement phases. As was already mentioned, the principal deployment site was in Kiruna, Sweden (see Figure 1 to view the operational region of SOLVE/THESEO 2000).

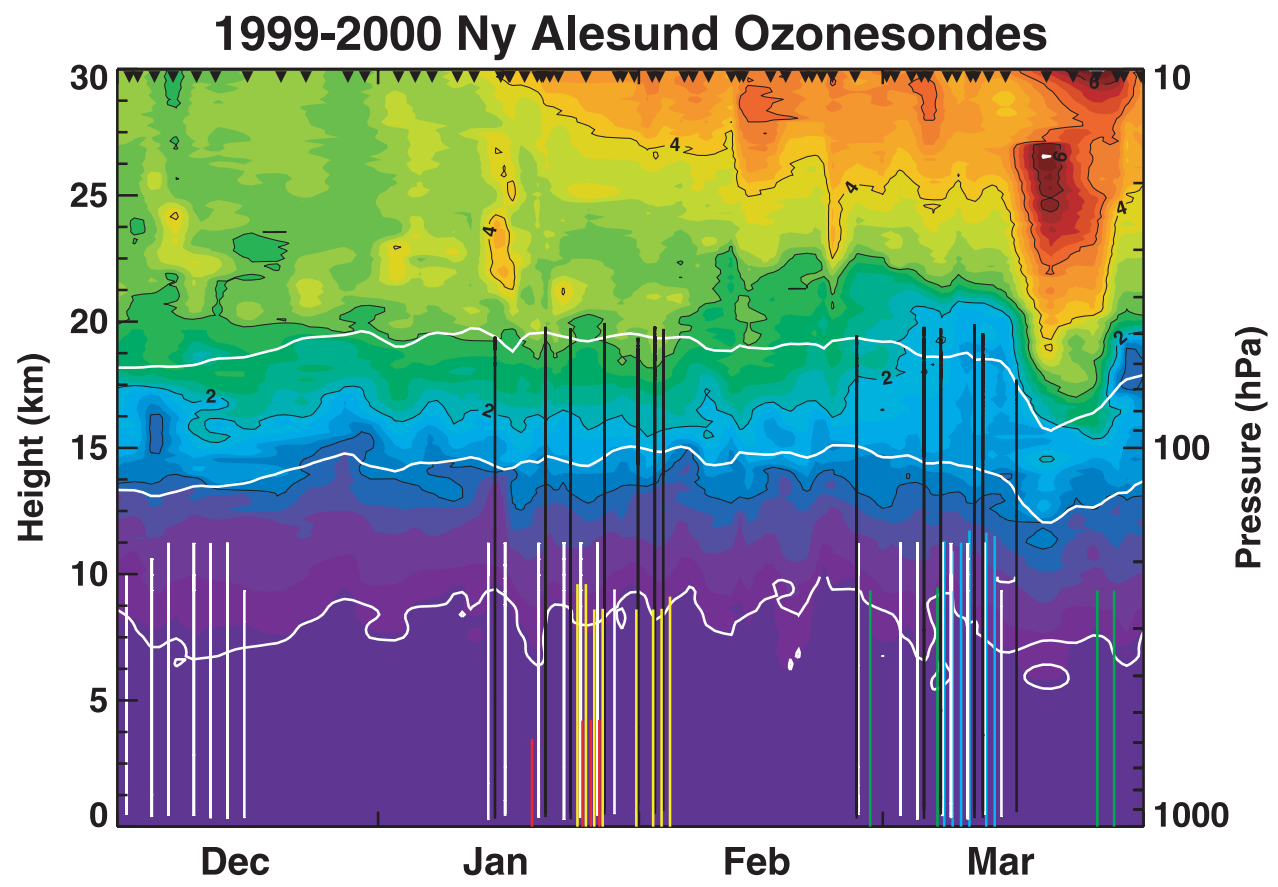

Figure 8. Ozone mixing ratios (ppmv) from ozonesonde measurements at Ny-Ålesund. The bottom white line indicates the tropopause, the middle white line is the $380 \mathrm{~K}$ isentropic level, and the top white line is the $480 \mathrm{~K}$ level. The vertical lines represent the altitude coverage of the DC-8 (white), ER-2 (black), Falcon (orange), ARAT (red), Mystère (green), and Lear jet (cyan) for their respective flight dates. The campaign phases are denoted by the active flying periods. 
Table 6a. SOLVE/THESEO 2000 Flight Log: Phase 1

\begin{tabular}{|c|c|c|c|c|c|c|c|}
\hline Date in 1999 & Platform & $\begin{array}{c}\text { Flight } \\
\text { Time }\end{array}$ & $\begin{array}{c}\text { Minimum } \\
\text { Latitude }\end{array}$ & $\begin{array}{c}\text { Maximum } \\
\text { Latitude }\end{array}$ & Site & Comment & Max. alt. MA $/ \mathrm{P} / \theta^{\mathrm{a}}$ \\
\hline 9 Nov. & DESCARTES & & & & ESRANGE & & \\
\hline 17 Nov. & $\begin{array}{l}\text { SAOZ } \\
\text { DESCARTES }\end{array}$ & & & & Andøya & & $27.0 \mathrm{~km} / 14.1 \mathrm{hPa} / 682.4 \mathrm{~K}$ \\
\hline 19 Nov. & OMS in situ & & & & ESRANGE & & $30.1 \mathrm{~km} / 8.14 \mathrm{hPa} / 789 \mathrm{~K}$ \\
\hline 30 Nov. & DC-8 & 9.90 & 34.87 & 78.07 & Kiruna & transit & $11.3 \mathrm{~km} / 216 \mathrm{hPa} / 347 \mathrm{~K}$ \\
\hline 2 Dec. & DC-8 & 8.36 & 63.04 & 79.59 & Kiruna & vortex survey & $11.3 \mathrm{~km} / 216 \mathrm{hPa} / 337 \mathrm{~K}$ \\
\hline 3 Dec. & $\begin{array}{l}\text { OMS remote, } \\
\text { DESCARTES }\end{array}$ & 6.03 & & & ESRANGE & & $33.2 \mathrm{~km} / 4.65 \mathrm{hPa} / 960 \mathrm{~K}$ \\
\hline 5 Dec. & DC-8 & 8.75 & 61.94 & 80.70 & Kiruna & PSC survey & $11.9 \mathrm{~km} / 196 \mathrm{hPa} / 346 \mathrm{~K}$ \\
\hline 7 Dec. & DC-8 & 9.73 & 67.81 & 85.67 & Kiruna & PSC survey & $12.5 \mathrm{~km} / 178 \mathrm{hPa} / 359 \mathrm{~K}$ \\
\hline 10 Dec. & DC-8 & 8.53 & 67.51 & 82.83 & Kiruna & vortex survey & $12.5 \mathrm{~km} / 178 \mathrm{hPa} / 356 \mathrm{~K}$ \\
\hline 12 Dec. & DC-8 & 9.66 & 67.67 & 81.27 & Kiruna & PSC survey & $12.5 \mathrm{~km} / 178 \mathrm{hPa} / 360 \mathrm{~K}$ \\
\hline 14 Dec. & DC-8 & 8.33 & 59.62 & 69.02 & Kiruna & vortex survey & $12.5 \mathrm{~km} / 178 \mathrm{hPa} / 357 \mathrm{~K}$ \\
\hline 15 Dec. & DESCARTES & & & & ESRANGE & & $25.86 \mathrm{~km} /$ \\
\hline 16 Dec. & DC-8 & 10.48 & 34.91 & 78.18 & Kiruna & transit & $11.9 \mathrm{~km} / 196 \mathrm{hPa} / 352 \mathrm{~K}$ \\
\hline
\end{tabular}

${ }^{\mathrm{a}}$ Maximum altitude/pressure/temperature.

Table 6b. SOLVE/THESEO Flight Log: Phase $2^{\mathrm{b}}$

\begin{tabular}{|c|c|c|c|c|c|c|c|}
\hline Date in 2000 & Platform & $\begin{array}{l}\text { Flight } \\
\text { Time }\end{array}$ & $\begin{array}{l}\text { Minimum } \\
\text { Latitude }\end{array}$ & $\begin{array}{l}\text { Maximum } \\
\text { Latitude }\end{array}$ & Site & Comment & Max. alt. MA/P/ $/ \theta^{b}$ \\
\hline 14 Jan. & DC-8 & 10.00 & 34.88 & 78.18 & Kiruna & transit & $12.5 \mathrm{~km} / 178 \mathrm{hPa} / 347 \mathrm{~K}$ \\
\hline 14 Jan. & ER-2 & 7.30 & 42.20 & 71.72 & Kiruna & transit & $20.5 \mathrm{~km} / 51 \mathrm{hPa} / 484 \mathrm{~K}$ \\
\hline 16 Jan. & DC-8 & 10.04 & 60.15 & 82.00 & Kiruna & PSC survey & $12.5 \mathrm{~km} / 178 \mathrm{hPa} / 366 \mathrm{~K}$ \\
\hline 19 Jan. & $\begin{array}{l}\text { HALOZ1, } \\
\text { DIRAC }\end{array}$ & 1.8 & 67.6 & 67.6 & ESRANGE & PSC flight & 27 km/12.7 hPa/700 K \\
\hline 19 Jan. & PSC-analysis & $13,768 \mathrm{~s}$ & & & ESRANGE & & $17.4 \mathrm{hPa}$ \\
\hline 19 Jan. & ARAT & 2.05 & 62.22 & 67.81 & Kiruna & transit & $4.1 \mathrm{~km} / 586 \mathrm{hPa}$ \\
\hline $20 \mathrm{Jan}$. & DC-8 & 9.77 & 62.20 & 89.35 & Kiruna & PSC survey & $12.5 \mathrm{~km} / 178 \mathrm{hPa} / 347 \mathrm{~K}$ \\
\hline $20 \mathrm{Jan}$. & ER-2 & 8.04 & 65.26 & 89.11 & Kiruna & PSC survey & $20.9 \mathrm{~km} / 48 \mathrm{hPa} / 463 \mathrm{~K}$ \\
\hline 23 Jan. & DC-8 & 10.32 & 58.57 & 73.77 & Kiruna & vortex sunrise & $12.5 \mathrm{~km} / 178 \mathrm{hPa} / 343 \mathrm{~K}$ \\
\hline 23 Jan. & ER-2 & 5.65 & 58.727 & 3.86 & Kiruna & vortex sunrise & $20.8 \mathrm{~km} / 48 \mathrm{hPa} / 466 \mathrm{~K}$ \\
\hline 23 Jan. & Salomon & & 66 (avg.) & & ESRANGE & & $18.2 \mathrm{~km} / 59 \mathrm{hPa}$ \\
\hline 24 Jan. & Falcon & 1.53 & 56.57 & 67.26 & Kiruna & transit & $11.9 \mathrm{~km} / 229 \mathrm{hPa}$ \\
\hline 25 Jan. & DC-8 & 10.36 & 59.37 & 75.89 & Kiruna & mountain waves & $12.5 \mathrm{~km} / 177 \mathrm{hPa} / 346 \mathrm{~K}$ \\
\hline $25 \mathrm{Jan}$. & Falcon & 2.12 & 64.73 & 68.88 & Kiruna & mountain waves & $11.9 \mathrm{~km} / 229 \mathrm{hPa}$ \\
\hline 25 Jan. & micro-Radibal & & & & ESRANGE & & $21.0 \mathrm{~km} / 38 \mathrm{hPa}$ \\
\hline 25 Jan. & PSC-analysis & $13,044 \mathrm{~s}$ & & & ESRANGE & PSC flight & $24.6 \mathrm{hPa}$ \\
\hline 25 Jan. & ARAT & 1.53 & 67.69 & 68.74 & Kiruna & PSC analysis & $4.9 \mathrm{~km} / 525 \mathrm{hPa} / 299 \mathrm{~K}$ \\
\hline 25 Jan. & ARAT & 2.37 & 66.16 & 67.97 & Kiruna & PSC analysis & $4.9 \mathrm{~km} / 525 \mathrm{hPa} / 299 \mathrm{~K}$ \\
\hline 26 Jan. & ARAT & 2.25 & 67.05 & 68.25 & Kiruna & PSC survey & $4.8 \mathrm{~km} / 522 \mathrm{hPa} / 285 \mathrm{~K}$ \\
\hline 26 Jan. & Falcon & 1.47 & 62.15 & 68.18 & Kiruna & mountrain waves & $10.7 \mathrm{~km} / 267 \mathrm{hPa}$ \\
\hline 27 Jan. & TRIPLE & $16,586 \mathrm{~s}$ & & & ESRANGE & & $25.69 \mathrm{~km} / 18.94 \mathrm{hPa} / 618 \mathrm{~K}$ \\
\hline 27 Jan. & $\begin{array}{l}\text { HALOZ2 } \\
\text { DIRAC }\end{array}$ & 2.0 & & & ESRANGE & sunset & $26 \mathrm{~km} / 15.6 \mathrm{hPa} / 690 \mathrm{~K}$ \\
\hline 27 Jan. & Falcon & 1.33 & 67.27 & 68.46 & Kiruna & mountain waves & $10.7 \mathrm{~km} / 266 \mathrm{hPa}$ \\
\hline 27 Jan. & ARAT & 2.93 & 58.01 & 67.81 & Kiruna & transit & $4.8 \mathrm{~km} / 523 \mathrm{hPa} / 283 \mathrm{~K}$ \\
\hline 27 Jan. & DC-8 & 9.71 & 51.48 & 68.00 & Kiruna & edge survey & $12.5 \mathrm{~km} / 178 \mathrm{hPa} / 344 \mathrm{~K}$ \\
\hline 27 Jan. & ER-2 & 6.10 & 51.52 & 67.92 & Kiruna & edge survey & $21.0 \mathrm{~km} / 47 \mathrm{hPa} / 475 \mathrm{~K}$ \\
\hline 28 Jan. & $\begin{array}{l}\text { SAOZ } \\
\text { SAOZ-BrO, } \\
\text { DESCARTES, } \\
\mathrm{CH}_{4} \mathrm{O}_{3} \text { sensor }\end{array}$ & & & & ESRANGE & & $27 \mathrm{~km} / 12.7 \mathrm{hPa} / 754 \mathrm{~K}$ \\
\hline 29 Jan. & DC-8 & 10.78 & 34.91 & 78.18 & Kiruna & transit & $12.5 \mathrm{~km} / 178 \mathrm{hPa} / 368 \mathrm{~K}$ \\
\hline 31 Jan. & ER-2 & 3.90 & 67.81 & 79.05 & Kiruna & vortex survey & $20.4 \mathrm{~km} / 51 \mathrm{hPa} / 451 \mathrm{~K}$ \\
\hline 31 Jan. & Falcon & 2.56 & 68.98 & 79.21 & Kiruna & NAT-rocks & $10.7 \mathrm{~km} / 267 \mathrm{hPa}$ \\
\hline 2 Feb. & ER-2 & 7.57 & 64.19 & 77.86 & Kiruna & vortex survey & 20.9 km/48h Pa/459 K \\
\hline 2 Feb. & Falcon & 2.58 & 68.24 & 79.39 & Kiruna & NAT-rocks & $10.7 \mathrm{~km} / 267 \mathrm{hPa}$ \\
\hline 3 Feb. & ER-2 & 3.21 & 67.83 & 73.16 & Kiruna & multiple level & $20.8 \mathrm{~km} / 49 \mathrm{hPa} / 458 \mathrm{~K}$ \\
\hline 3 Feb. & Falcon & 1.44 & 68.45 & 73.39 & Kiruna & NAT-rocks & $10.7 \mathrm{~km} / 266 \mathrm{hPa}$ \\
\hline 4 Feb. & Falcon & 2.28 & 48.76 & 66.35 & Kiruna & transit & $11.3 \mathrm{~km} / 247 \mathrm{hPa}$ \\
\hline 9 Feb. & $\begin{array}{l}\mathrm{SAOZ}, \\
\text { SAOZ-BrO, } \\
\mathrm{CH}_{4} \mathrm{O}_{3} \text { sensor }\end{array}$ & & & & ESRANGE & & $25 \mathrm{~km} / 21.4 \mathrm{hPa} / 632 \mathrm{~K}$ \\
\hline 13 Feb. & $\begin{array}{l}\text { SAOZ, } \\
\text { SAOZ-BrO } \\
\text { DESCARTES, } \\
\mathrm{CH}_{4} \mathrm{O}_{3} \text { sensor }\end{array}$ & & & & ESRANGE & & $26 \mathrm{~km} / 17.9 \mathrm{hPa} / 729 \mathrm{~K}$ \\
\hline
\end{tabular}

${ }^{\mathrm{a}}$ In addition, NOAA/CMDL frost point hygrometers were launched as separate payloads on 10 November (coinciding with OMS), 14 November, 20 November, 26 November, 25 January (after PSC Analysis), 27 January (after Triple and Haloz), 3 March, and 5 March (with OMS).

${ }^{\mathrm{b}}$ Maximum altitude/pressure/temperature. 
Table 6c. SOLVE/THESEO Flight Log: Phase 3

\begin{tabular}{|c|c|c|c|c|c|c|c|}
\hline Date in 2000 & Platform & $\begin{array}{l}\text { Flight } \\
\text { time }\end{array}$ & $\begin{array}{l}\text { Minimum } \\
\text { Latitude }\end{array}$ & $\begin{array}{l}\text { Maximum } \\
\text { Latitude }\end{array}$ & Site & Comment & Max. alt. MA/P/ $/ \theta^{\mathrm{a}}$ \\
\hline 18 Feb. & LPMA/DOAS & & & & ESRANGE & & $29.5 \mathrm{~km}$ \\
\hline 18 Feb. & MIR 1e & & & & ESRANGE & 2 days & \\
\hline 18 Feb. & MIR 2e & & & & ESRANGE & 18 days & \\
\hline 22 Feb. & Salomon $2 \mathrm{e}$ & & & & ESRANGE & & $29 \mathrm{~km} / 11.4 \mathrm{hPa} / \mathrm{NA}$ \\
\hline 26 Feb. & ER-2 & 7.46 & 62.93 & 84.80 & Kiruna & vortex survey & $20.5 \mathrm{~km} / 50 \mathrm{hPa} / 455 \mathrm{~K}$ \\
\hline 27 Feb. & $\mathrm{SAOZ}, \mathrm{CH}_{4}, \mathrm{LABS}$ & & & & ESRANGE & MIR support & $24 \mathrm{~km} / 22 \mathrm{hPa} / 632 \mathrm{~K}$ \\
\hline 27 Feb. & DC-8 & 10.25 & 34.88 & 78.18 & Kiruna & transit & $12.5 \mathrm{~km} / 179 \mathrm{hPa} / 352 \mathrm{~K}$ \\
\hline 28 Feb. & Mystère 20 & 2.4 & 45.75 & 49.16 & Paris & circular & $10.7 \mathrm{~km} / 238 \mathrm{hPa} / 330 \mathrm{~K}$ \\
\hline 29 Feb. & techno. MIR 3 & & & & ESRANGE & & \\
\hline $1 \mathrm{March}$ & TRIPLE & & & & ESRANGE & & $21.7 \mathrm{hPa} / \mathrm{NA}$ \\
\hline $1 \mathrm{March}$ & HALOZ3, DESCARTES & 1.8 & 67.5 & 67.6 & ESRANGE & ozone loss & $25 \mathrm{~km} / 18 \mathrm{hPa} / 665 \mathrm{~K}$ \\
\hline 3 March & DC-8 & 9.99 & 60.69 & 86.53 & Kiruna & vortex edge & $12.5 \mathrm{~km} / 178 \mathrm{hPa} / 338 \mathrm{~K}$ \\
\hline 5 March & DC-8 & 9.55 & 63.82 & 79.37 & Kiruna & vortex survey & $12.5 \mathrm{~km} / 178 \mathrm{hPa} / 337 \mathrm{~K}$ \\
\hline 5 March & ER-2 & 7.85 & 66.68 & 81.04 & Kiruna & vortex survey & $20.8 \mathrm{~km} / 48 \mathrm{hPa} / 467 \mathrm{~K}$ \\
\hline 5 March & $\begin{array}{l}\text { OMS in situ, DIRAC, } \\
\text { DESCARTES }\end{array}$ & & & & ESRANGE & & $29.3 \mathrm{~km} / 8.89 \mathrm{hPa} / 845 \mathrm{~K}$ \\
\hline 7 March & $\begin{array}{l}\text { SAOZ, SAOZ-BrO Labs, } \\
\text { DESCARTES }\end{array}$ & & & & ESRANGE & & $27 \mathrm{~km} / 9.7 \mathrm{hPa} / 820 \mathrm{~K}$ \\
\hline 7 March & Mystère 20 & 2.0 & 46.13 & 58.32 & Paris & & $10.8 \mathrm{~km} / 234 \mathrm{hPa} / 330 \mathrm{~K}$ \\
\hline $7 \mathrm{March}$ & ER-2 & 7.90 & 67.83 & 82.96 & Kiruna & vortex survey & $20.8 \mathrm{~km} / 48 \mathrm{hPa} / 467 \mathrm{~K}$ \\
\hline 8 March & DC-8 & 10.67 & 63.10 & 80.25 & Kiruna & PSC survey & $12.5 \mathrm{~km} / 178 \mathrm{hPa} / 336 \mathrm{~K}$ \\
\hline 8 March & HALOZ4, DIRAC & 1.8 & & & ESRANGE & ozone loss & $25 \mathrm{~km} / 19 \mathrm{hPa} / 670 \mathrm{~K}$ \\
\hline $8 \mathrm{March}$ & Lear jet & 3.05 & 49.42 & 68.12 & Kiruna & transit & 12.59 \\
\hline 9 March & DC-8 & 9.92 & 57.40 & 80.14 & Kiruna & & $12.5 \mathrm{~km} / 178 \mathrm{hPa} / 346 \mathrm{~K}$ \\
\hline 9 March-0 & Lear jet & 2.78 & 64.68 & 70.02 & Kiruna & Iceland flight & $11.537 \mathrm{~km}$ \\
\hline 9 March-1 & Lear jet & 2.31 & 64.81 & 70.02 & Kiruna & Iceland flight & $12.204 \mathrm{~km}$ \\
\hline 10 March-0 & Lear jet & 1.30 & 69.26 & 77.82 & Kiruna & North Pole & $11.744 \mathrm{~km}$ \\
\hline 10 March-1 & Lear jet & 3.08 & 79.06 & 89.95 & & North Pole & $12.526 \mathrm{~km}$ \\
\hline 10 March-2 & Lear jet & 0.90 & 68.61 & 75.15 & Kiruna & North Pole & $11.191 \mathrm{~km}$ \\
\hline 11 March & Lear jet & 3.5 & 68.22 & 76.03 & Kiruna & Arctic Sea & $12.995 \mathrm{~km}$ \\
\hline 11 March & DC-8 & 8.78 & 67.58 & 80.09 & Kiruna & vortex edge & $12.5 \mathrm{~km} / 178 \mathrm{hPa} / 342 \mathrm{~K}$ \\
\hline 11 March & ER-2 & 7.97 & 58.60 & 75.08 & Kiruna & vortex edge & $20.9 \mathrm{~km} / 47 \mathrm{hPa} / 474 \mathrm{~K}$ \\
\hline 12 March & ER-2 & 7.44 & 65.27 & 79.46 & Kiruna & vortex survey & $20.6 \mathrm{~km} / 50 \mathrm{hPa} / 466 \mathrm{~K}$ \\
\hline 13 March & DC-8 & 8.66 & 65.07 & 83.91 & Kiruna & vortex survey & $12.5 \mathrm{~km} / 178 \mathrm{hPa} / 354 \mathrm{~K}$ \\
\hline 13 March-0 & Lear jet & 3.32 & 48.03 & 69.66 & Kiruna & transit & $12.58 \mathrm{~km}$ \\
\hline 13 March-1 & Lear jet & 3.88 & 28.72 & 46.52 & Dübendorf & transit & $12.915 \mathrm{~km}$ \\
\hline 14 March & Lear jet & 4.14 & 29.13 & 47.37 & Canary Is. & transit & $12.781 \mathrm{~km}$ \\
\hline 15 March & DC-8 & 8.77 & 46.95 & 86.33 & Kiruna & transit & $11.3 \mathrm{~km} / 216 \mathrm{hPa} / 349 \mathrm{~K}$ \\
\hline 15 March & OMS remote & 5.01 & & & ESRANGE & & $29.1 \mathrm{~km} / 11.3 \mathrm{hPa} / 850 \mathrm{~K}$ \\
\hline 16 March & ER-2 & 9.02 & 42.21 & 67.81 & Kiruna & transit & $20.4 \mathrm{~km} / 51 \mathrm{hPa} / 508 \mathrm{~K}$ \\
\hline 25 March & $\begin{array}{l}\text { SAOZ, BrO. } \\
\text { DIRAC } \mathrm{CH}_{4}\end{array}$ & & & & ESRANGE & & $28 \mathrm{~km} / 12.3 \mathrm{hPa} / 837 \mathrm{~K}$ \\
\hline 26 March & Mystère 20 & 2.4 & 45.7 & 49.16 & Paris & circular & $10.7 \mathrm{~km} / 238 \mathrm{hPa} / 330 \mathrm{~K}$ \\
\hline $28 \mathrm{March}$ & Mystère 20 & 2.5 & 46.94 & 51.80 & Paris & & $10.7 \mathrm{~km} / 238 \mathrm{hPa} / 330 \mathrm{~K}$ \\
\hline 3 April & SAOZ, DESCARTES & & & & Andøya & & $26 \mathrm{~km} / 11.9 \mathrm{hPa} / 822 \mathrm{~K}$ \\
\hline 4 April & HALOZ5 DESCARTES & 1.5 & 67.6 & 67.8 & ESRANGE & low-altitude survery & $17 \mathrm{~km} / 78 \mathrm{hPa} / 420 \mathrm{~K}$ \\
\hline
\end{tabular}

${ }^{\text {a }}$ Maximum altitude/pressure/temperature. NA, not available.

Five of the aircraft were primarily based in Arena Arctica at the Kiruna Airport. The balloons were nearly all flown from Esrange, with two additional SAOZ balloons launched from Andøya, Norway (240 km northwest of Kiruna).

[56] The aircraft flights indicated in Figure 8 cluster into the three phases of the campaign. The balloon flights are shown in Figure 9, superimposed on the polar vortex average temperatures (the three thick black lines at the bottom of the figure indicate the campaign phases). The red line is the NAT PSC saturation temperature calculated from the average vortex temperature assuming $4.5 \mathrm{ppmv}$ of water vapor and $10 \mathrm{ppbv}$ of nitric acid [Hanson and Mauersberger, 1988]. This line gives a rough indication of when PSCs were present in significant quantities in the vortex but does not represent the appearance of small localized cold regions. As planned, the first phase sampled the polar vortex during the vortex formation phase temperatures cooled below the NAT saturation temperature of $195 \mathrm{~K}$. The NAT saturation temperature is near $195 \mathrm{~K}$ on the $500 \mathrm{~K}$ isentropic level and $190 \mathrm{~K}$ at the $600 \mathrm{~K}$ level. The second phase occurred shortly after the coldest period during the 1999-2000 winter in the last two weeks of January. The final phase occurred as temperatures warmed to temperatures greater than $195 \mathrm{~K}$.

\subsection{Mission Forecasting}

[57] The aircraft platforms and balloon payloads discussed in the previous sections provided an enormous capability for understanding the Arctic polar vortex. However, such resources required careful coordination and direction for optimizing the scientific return. The optimized flight paths were determined by using meteorological, chemical, and microphysical forecasting. A number of meteorological and theory groups participated in the SOLVE/THESEO 2000 campaign. Over the course of the campaign these groups held daily meetings at Arena Arctica and Esrange to discuss the evolving polar vortex and to forecast its further evolution. In addition, tropospheric 


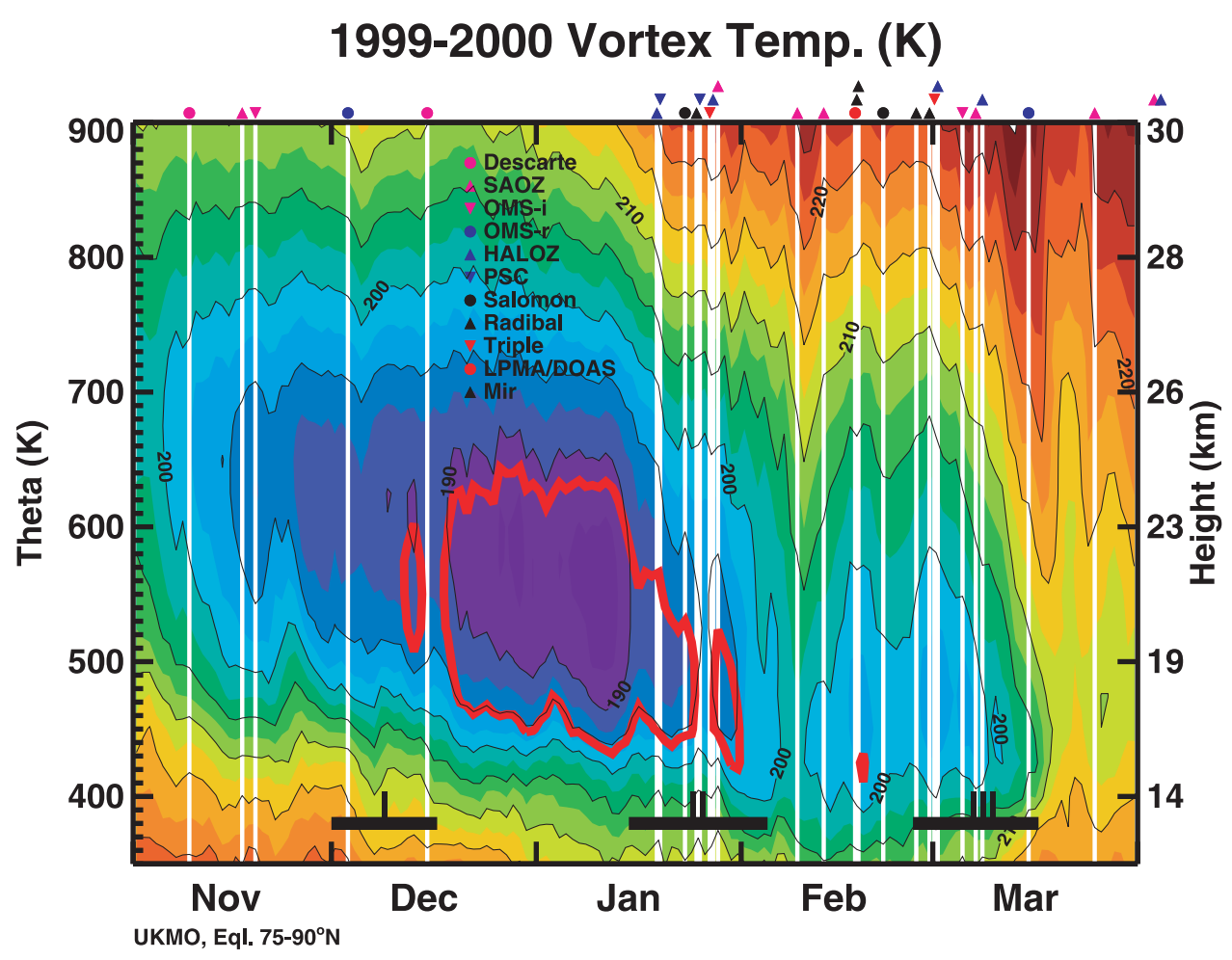

Figure 9. Average vortex temperature as a function of potential temperature. The height scale on the right axis is an estimate based upon a $6.5-\mathrm{km}$ scale height. The average is computed over an equivalent latitude range from $75^{\circ}$ to $90^{\circ} \mathrm{N}$ using the UKMO data at each theta level from 350 to $900 \mathrm{~K}$. The red line is the NAT PSC saturation temperature calculated from the average vortex temperature by using $4.5 \mathrm{ppmv}$ of water vapor and $10 \mathrm{ppbv}$ of nitric acid [Hanson and Mauersberger, 1988]. The white vertical lines indicate balloon launches, and symbols at the top indicate the balloon payload (see Tables $6 \mathrm{a}-6 \mathrm{c}$ and the payload section for details). Campaign phases are superimposed.

forecasts were used to determine flight conditions, particularly for the large balloon payloads and NASA ER-2.

\subsubsection{Tropospheric Forecasting}

[58] The surface weather forecasting for SOLVE/THESEO 2000 at the Arena Arctica was conducted by G. Forbes (The Weather Channel). Forecasting for the DC-8, ARAT, Falcon, and Lear jet was relatively straightforward, since these aircraft have a near "all-weather" flight capability. However, the NASA ER-2 has rather severe weather restrictions. For example, the ER-2 requires runway cross winds to be less than 12 knots $(6 \mathrm{~m} / \mathrm{s})$, wind chill temperatures greater than $-31^{\circ} \mathrm{C}$, good visibility, low ceilings, and a runway that is relatively free of ice and snow. Such conditions were satisfied relatively often in Kiruna but were extremely difficult to forecast.

[59] Surface forecasting at Esrange was handled by the U.S. National Science Balloon Facility (NSBF) staff meteorologists (G. Rosenberger, J. Hobbie, and R. Mullenax) for the SOLVE OMS launches and by CNES meteorologists (P. Dedieu and colleagues) for the THESEO 2000 launches. For larger balloons, very light surface winds and very small vertical wind shears over the lowest few hundred meters are necessary for safe launches. Smaller balloons could be launched under less favorable conditions. Forecasting support was also provided by Esrange staff, and sounding balloon launches were handled by the Esrange balloon launch crew. Trajectory forecasts were an important aspect of the balloon launches. These trajectories ensured that the payloads were inside the vortex, had the desired float times, and could be brought down in a region convenient and safe for recovery. For example, the second OMS remote launch required very accurate predictions of time to float (a heavy cirrus cover caused an unfavorable radiation environment). The NSBF meteorologists' use of the SINBAD model was critical in determining the launch window. Similarly, the accurate ground wind forecasts from the Météo-France Arpège model and from SMHI were essential in the launch of the first PSC-Analysis gondola. All of the trajectory and meteorological forecasts were prepared with considerable assistance by the Esrange Range Safety team (T. Hedqvist, S. Kemi, and B. Sjoholm).

\subsubsection{Stratospheric Forecasting}

[60] The forecasting for the stratosphere was carried out by a number of groups. These groups used standard meteorological analyses combined with specialized products to predict stratospheric temperatures, the position of the polar vortex, mountain wave amplitudes and turbulence, PSC microphysics, and the chemical composition of the stratosphere. Table 7 lists the analyses or models with the PI or group that was responsible for both supplying the data to the field, and analyzing those data.

\subsubsection{Stratospheric Meteorology and Mountain Wave} Forecasting

[61] A number of conventional meteorological forecasts and analyses were utilized for SOLVE/THESEO 2000 forecasting. These forecasts and analyses were routinely 
Table 7. Analysis, Forecasts, and Modeling

\begin{tabular}{|c|c|c|c|}
\hline $\begin{array}{c}\text { Analysis or } \\
\text { Model }\end{array}$ & Investigator & Institution & Measurements or Outpu \\
\hline $\begin{array}{l}\text { DAO GEOS } 3 \text { analyses } \\
\text { and forecasts }\end{array}$ & S. Strahan & $\begin{array}{l}\text { Univ. of Maryland } \\
\text { Baltimore County }\end{array}$ & $\mathrm{U}, \mathrm{V}, \mathrm{Z}, \mathrm{T}, \mathrm{Pv}$ \\
\hline $\begin{array}{l}\text { NCEP/CPC analyses } \\
\text { NCEP/MRF forecasts } \\
\text { and analyses }\end{array}$ & L. Lait & SSAI & $\begin{array}{c}\mathrm{U}, \mathrm{V}, \mathrm{Z}, \mathrm{T}, \mathrm{Pv}, \\
\text { trajectories }\end{array}$ \\
\hline NCEP/AVN & A. Tuck & NOAA/AL & $\mathrm{U}, \mathrm{V}, \mathrm{T}$ \\
\hline $\begin{array}{l}\text { Australian GASP analyses } \\
\text { and forecasts }\end{array}$ & D. Waugh & Johns Hopkins Univ. & $\mathrm{U}, \mathrm{V}, \mathrm{T}$, trajectories \\
\hline $\begin{array}{l}\text { ECMWF analyses } \\
\text { and forecasts }\end{array}$ & $\begin{array}{l}\text { G. Braathen } \\
\text { A. Dörnbrack } \\
\text { B. Knudsen }\end{array}$ & $\begin{array}{l}\text { NILU } \\
\text { DLR } \\
\text { DMI }\end{array}$ & $\mathrm{U}, \mathrm{V}, \mathrm{Z}, \mathrm{T}, \mathrm{PV}$ \\
\hline $\begin{array}{l}\text { Free U. of Berlin analyses } \\
\text { NCEP/CPC analyses }\end{array}$ & $\begin{array}{l}\text { B. Naujokat } \\
\text { H. Selkirk }\end{array}$ & $\begin{array}{l}\text { Free Univ. of Berlin } \\
\text { NASA/ARC }\end{array}$ & $\begin{array}{l}\mathrm{Z}, \mathrm{T} \\
\text { trajectories }\end{array}$ \\
\hline $\begin{array}{l}\text { NCEP/MRF forecasts } \\
\text { and analyses }\end{array}$ & & & \\
\hline Mimosa model & A. Hauchecorne & CNRS-SA/IPSL & trajectories \\
\hline $\begin{array}{l}\text { CLaMS - Chemical } \\
\text { Lagrangian Model }\end{array}$ & R. Müller & FZ Jülich & trajectories \\
\hline Match & M. Rex & AWI & trajectories \\
\hline MWFM & S. Eckerman & NRL & $\begin{array}{l}\text { mountain wave } \mathrm{T} \text {, } \\
\text { amplitude, turbulence }\end{array}$ \\
\hline DLR MM5 & A. Dörnbrack & DLR & mesoscale flow fields \\
\hline HRM Model & H. Wernle & ETH-Zürich & mesoscale flow fields \\
\hline $\begin{array}{l}\text { Univ. of Wisconsin 3-D } \\
\text { nonhydrostatic model }\end{array}$ & M. Hitchman & Univ. of Wisconsin & $\begin{array}{l}\text { mesoscale to synoptic } \\
\text { scale flow fields }\end{array}$ \\
\hline 3-D VOM & S. Vosper & Univ. of Leeds & mesoscale flow fields \\
\hline GSFC 3-D CTM & R. Kawa & NASA/GSFC & $\begin{array}{l}\text { source, reservoir, and } \\
\text { radical species, } \\
\text { chemical trajectories }\end{array}$ \\
\hline SLIMCAT & $\begin{array}{l}\text { M. Chipperfield } \\
\text { J. Pyle }\end{array}$ & $\begin{array}{l}\text { Univ. of Leeds } \\
\text { Univ. of Cambridge }\end{array}$ & $\begin{array}{l}\text { source, reservoir, and } \\
\text { radical species }\end{array}$ \\
\hline REPROBUS & F. Lefevre & CNRS-SA & $\begin{array}{l}\text { source, reservoir, and } \\
\text { radical species }\end{array}$ \\
\hline IMPACT & K. Drdla & NASA/ARC & $\begin{array}{l}\text { chemical trajectories, } \\
\text { microphysics }\end{array}$ \\
\hline $\begin{array}{l}\text { Photochemical } \\
\text { steady state (PSS) }\end{array}$ & R. Salawitch & JPL & radical species \\
\hline Photolysis rates & S. Lloyd & JHU/APL & $J$ values \\
\hline
\end{tabular}

provided for flight planning and data analysis. Typically, the data were used to forecast the locations of cold temperature regions (for PSC flights) and for locating the position of the polar vortex. These analyses included GSFC Data Assimilation Office (DAO) GEOS 3 output, the NCEP/CPC analyses and NCEP/MRF forecasts and analyses, the NCEP/ AVN output, the Australian Bureau of Meteorology's Global Assimilation and Prediction (GASP) system's analyses and forecasts, and the ECMWF forecasts and analyses. The Météo-France Arpège model was used for stratospheric as well as tropospheric forecasting. The UKMO UARS analyses were also used to support the analysis of SOLVE/ THESEO 2000 observations. Free University of Berlin subjective analyses of radiosondes were provided for analysis purposes.

[62] A number of specialized products were utilized for forecasting and analysis. High-density trajectory model runs based on analyzed and forecast winds were used to predict air masses that had broken off of the polar vortex, had encountered PSCs, had persistently been in polar darkness, or had encountered upper tropospheric clouds. Such trajectory runs based on forecast and analysis products were produced by using the NCEP and DAO forecasts, the DAO data in combination with NCEP/MRF analyses in the upper troposphere and lower stratosphere for studying water vapor transport, the GASP data, and the MIMOSA model at
CNRS. Vortex filaments were also forecast by using the Chemical Lagrangian Model of the Stratosphere (CLaMS). [63] The Match project [Rex et al., 2002] provided realtime trajectory analyses during the campaign. The main goal was to track and to forecast the motions of air masses that have been probed by ozonesondes and to enable subsequent ozone measurements within these air masses in a Lagrangian sense. Close coincident measurements of ozonesondes with the ER-2 instruments were also coordinated on the basis of the ER-2 flight plans and short trajectory forecasts of the motions of the air masses probed along the envisaged flight track. After each ER-2 flight the motions of air masses probed along the actual flight track were tracked and forecast by continuously updated trajectory calculations over 10 days. The positions of the air masses probed during previous flights were made available for flight planning and for the coordination of Lagrangian ozonesonde measurements.

[64] Mountain-forced gravity waves are extremely important for understanding the evolution and formation of PSCs but also for forecasting turbulence that posed a hazard to the NASA ER-2. Forecasts of mountain wave vertical displacement amplitudes, temperature amplitudes, and turbulence were provided by using the Naval Research Laboratory Mountain Wave Forecast Model (MWFM) interfaced to forecast winds and temperatures from the DAO GEOS 2 
forecasts and the U.S. Navy's NOGAP model forecasts. Flow fields and temperature perturbations for the whole of Scandinavia were provided on the basis of the regional MM5 model and the similar HRM model. In addition, the HRM model was used to provide forecast trajectories for the Triple payload (see section 2.2.4.6). The three-dimensional (3-D) University of Wisconsin Non-hydrostatic Modeling System (UW-NMS) was used to investigate transport processes at the mesoscale to synoptic scale in the upper troposphere/lower stratosphere near Scandinavia. The 3DVOM is a finite difference numerical code designed for high-resolution simulations. Finally, the flow fields and temperature perturbation over northern Scandanavia were also calculated by using the $15-\mathrm{km}$ resolution German Weather Service NWP model.

\subsubsection{Chemical and Radiative Modeling}

[65] While the meteorology provided the broad strategy for the campaign, chemical and radiative models provided supplemental information for designing flight paths. Three 3-D chemical transport models (CTM) were employed during SOLVE/THESEO 2000. The GSFC CTM uses DAO GEOS 2 meteorology to provide a full suite of trace gas measurements, including source gases, reservoir species, and radical species in all of the major families. The UK UGAMP SLIMCAT model is an isentropic 3-D CTM that uses UKMO meteorology to provide a full suite of trace gases. The REPROBUS model is a full 3-D CTM that uses the Météo-France Arpège analysis and forecast winds to produce a complete set of stratospheric species.

[66] In addition to the 3-D models, a chemical trajectory model was run for each of the ER-2 flights and for parcels within the polar vortex using the GSFC trajectory model. A photochemical steady state model was heavily utilized for forecasting the behavior of radical species under a variety of conditions. A radiative transfer model was extensively used to provide photolysis rate coefficients or $j$ values for photochemical reactions of interest in the lower stratosphere and upper troposphere, calculated along the ER-2 and DC-8 flight tracks for each flight.

\subsubsection{Microphysical Modeling}

[67] Determining the evolution and properties of PSCs was a principal goal of the SOLVE/THESEO 2000 campaign. While temperature forecasts were the principal tools for locating prospective regions of PSC formation, microphysical models were employed for real-time analysis of the observations in order to redirect our strategies while we were in the field. The Integrated Microphysics and Aerosol Chemistry on Trajectories (IMPACT) model was used to simulate PSCs and their impact on stratospheric chemistry. The MM5 results from the DLR and the HRM trajectories were used with the Mainz microphysical box model [Meilinger et al., 1995; Tsias et al., 1997; Carslaw et al., 1998] for calculation of PSC temporal evolution along air parcel trajectories.

\section{Results}

[68] As was described in section 2.1, the SOLVE/THESEO 2000 campaign was partitioned into three phases: (1) the early December polar vortex setup period; (2) the very cold, late January period; and (3) the vortex recovery period. As will be shown, all three phases were adequately covered, as had been hoped. Ground-based, satellite, and ozonesonde measurements provided continuous coverage throughout the winter and filled the observational gaps between the phases.

[69] The first phase of SOLVE/THESEO 2000 was timed to occur in early winter as temperatures cooled to the point where PSCs would form. Balloon flights and the NASA DC-8 flights were prioritized to understand the initial conditions of the polar vortex prior to the occurrence of significant PSCs and to measure some of the first appearances of PSCs. Initial flights of the DC-8 and the OMS remote payload did not reveal elevated levels of $\mathrm{ClO}$, suggesting that the first goal was achieved [Bremer et al., 2002]. In addition, a number of early PSCs were detected by the DC-8 in flights over the high Arctic during the course of the first phase.

[70] The second phase was planned to occur in midwinter, when temperatures were at their lowest. In reality, the aircraft and balloon flights were all conducted shortly after the coldest period in the 1999-2000 winter. All of the flights inside the polar vortex encountered air that had been chemically perturbed by PSCs. In addition, vast regions of the polar vortex were still cold enough in this late January period to contain PSCs.

[71] The final campaign phase was set for the first two weeks of March to investigate the recovery of the polar vortex. Surprisingly, at the start of this phase the polar vortex was quite strong and temperatures were still cold enough to form PSCs. Again, both chemically perturbed air and PSCs were measured inside the polar vortex during this phase.

\subsection{Meteorological Background}

[72] The early stratospheric winter of 1999-2000 has been described by Manney and Sabutis [2000]. In this paper they point out the unusually cold early winter lower stratosphere and describe the vortex evolution in 1999-2000 as typical. The breakup of the 1999-2000 lower stratospheric vortex occurred on 11 April 2000, slightly later than the average breakup date of 29 March [Waugh and Rong, 2002].

\subsubsection{Temperatures}

[73] The temperature of the polar vortex over the course of the 1999-2000 winter has been shown in Figure 1 of Manney and Sabutis [2000]. The vortex average temperature is shown in Figure 9 of this paper with all of the SOLVE/THESEO 2000 balloon flights. The first balloon flights and the DC-8 flights occurred as the lower stratospheric vortex was cooling below $195 \mathrm{~K}$ during the first phase of the campaign. Lidar temperature observations inside the vortex, ozonesondes, and conventional radiosondes all provided direct evidence of this cold polar vortex in the middle-to-lower stratosphere of the Arctic during this early winter phase.

[74] Figure 9 shows that the coldest temperatures occurred in the first two weeks of January, immediately prior to the second phase of the campaign. Again, direct observations provide an independent validation of these cold lower stratospheric temperatures. Extensive measurements of PSCs were obtained during this second phase.

[75] During the final phase of SOLVE/THESEO 2000, the polar lower stratospheric temperatures increased above 195 K. Again, Figure 9 displays this increase during the first 


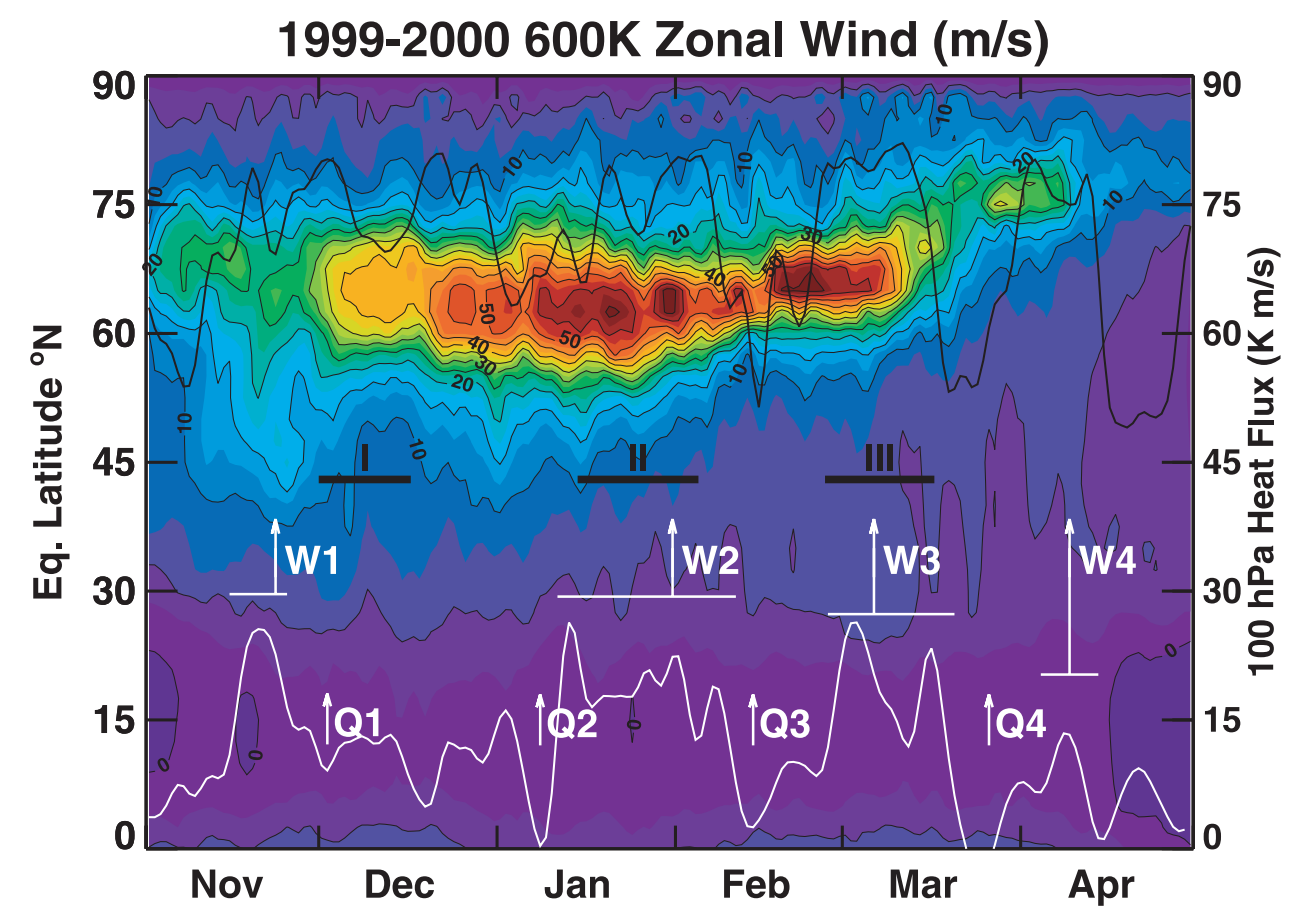

Figure 10. Mean wind parallel to lines of equivalent latitude on the $600 \mathrm{~K}$ isentropic surface (equivalent to a zonal mean in a vortex-centered coordinate system). Also shown are the equivalent latitude of Kiruna (black line) and the eddy heat flux of large-scale waves at $100 \mathrm{hPa}$ (white line). Campaign phases are superimposed.

two weeks of March. The minimum temperature of the vortex was colder than shown herein because Figure 9 is a vortex average. PSCs were observed over extensive regions during this third phase. During the final flights of the DC-8 from Kiruna during this phase, temperatures were observed to be too warm for the formation of PSCs. The last PSC observed by the POAM satellite instrument occurred on 15 March.

\subsubsection{Vortex and Jet Evolution}

[76] The lower stratospheric polar vortex developed slowly during the 1999-2000 winter [Manney and Sabutis, 2000], reached its greatest strength in mid-January, and broke apart in mid-April [Waugh and Rong, 2002]. This evolution is illustrated in Figure 10, which displays the average wind on the $600 \mathrm{~K}$ isentropic surface following lines of equivalent latitude (equivalent to a zonal mean in a vortex-centered coordinate system). In the midstratosphere, the polar night jet core speed was greater than $20 \mathrm{~m} / \mathrm{s}$ by early November, $30 \mathrm{~m} / \mathrm{s}$ by early December, and $50 \mathrm{~m} / \mathrm{s}$ by late December. The polar vortex is the region contained by this polar night jet.

[77] This jet had three periods of deceleration: late January to early February as the jet narrowed in latitudinal dimension (indicated by W2 in Figure 10), mid-March as the jet decelerated and the polar vortex shrank (W3 in Figure 10), and early April as a greatly reduced vortex disappeared completely (W4 in Figure 10). The SOLVE/ THESEO 2000 phases are superimposed on Figure 10. Phase I coincided with the rapid vortex acceleration (Q1 in Figure 10). The second phase occurred as the vortex decelerated and narrowed (W2 in Figure 10), while the third phase occurred as the vortex was rapidly shrinking (W3 in Figure 10).
[78] Generally, Kiruna's equivalent latitude was poleward of the jet's core; hence Kiruna was usually inside the polar vortex over the course of the 1999-2000 winter. The thick black line in Figure 10 shows the equivalent latitude of Kiruna on this $600 \mathrm{~K}$ isentropic surface. Kiruna is outside the vortex during mid-February and late March (aside from the spinup and breakdown periods of the vortex). At lower stratospheric altitudes (e.g., $450 \mathrm{~K}$ ), the vortex is more variable, and Kiruna was found to be frequently outside of the vortex. Kiruna was inside the vortex during all three phases of the campaign.

[79] The behavior of the polar night jet in Figure 10 can be broadly explained by examining the wave energy propagating out of the troposphere and into the stratosphere over the course of the campaign. The large wave events act to decelerate the jet and warm the polar vortex [Newman et al., 2001]. The wave energy that drives the circulation of the stratosphere is proportional to the eddy heat flux for largescale waves (superimposed on Figure 10 as the white line; scale on the right-hand side of the figure). There are four important bursts of wave energy (large eddy heat flux) that affect the stratosphere during the 1999-2000 winter. The heat flux shown in Figure 10 has been smoothed to emphasize the week-to-week variability. The first burst (indicated by W1 in Figure 10) acts to retard both the jet development and the radiative cooling of the polar region. The jet builds and the polar region cools as this first event falls off. The second burst (W2 in Figure 10) acts to narrow the broad flow and warm the polar vortex from the cold early January temperatures. The third burst (indicated by W3) warms the vortex above temperatures necessary for the formation of polar stratospheric clouds and considerably reduces the jet speed and vortex size. The fourth burst in 

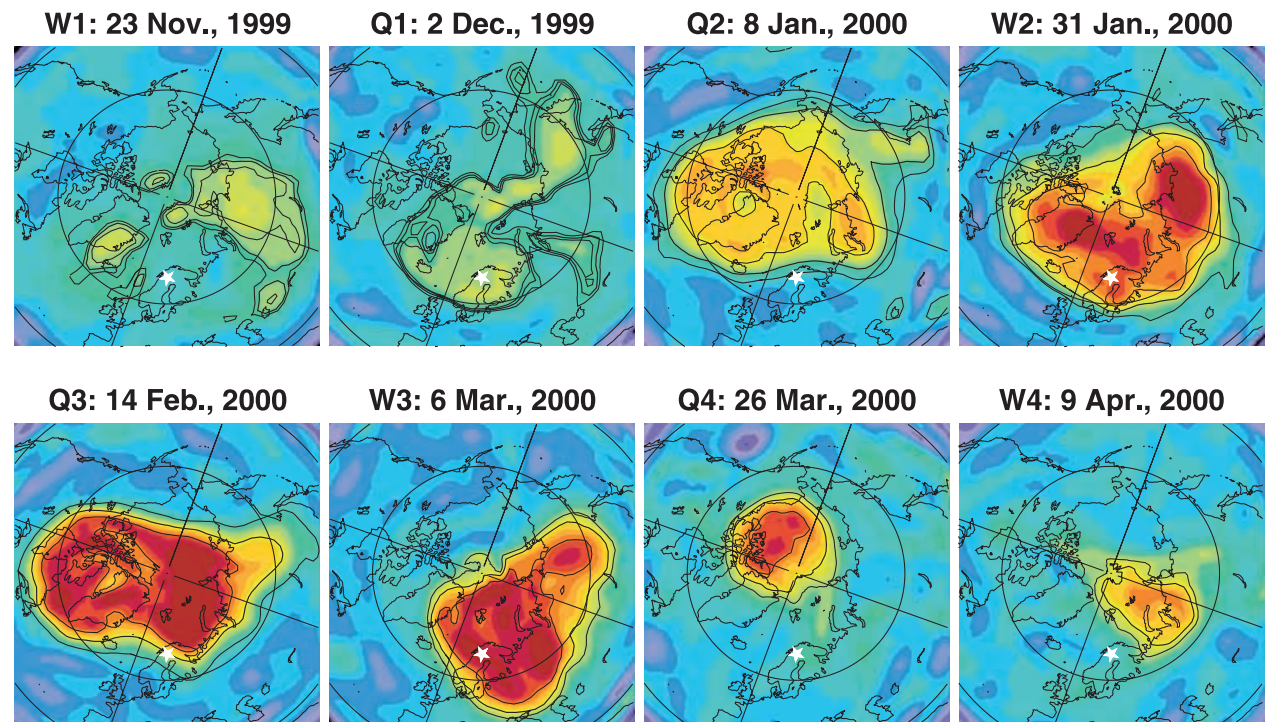

W4: 9 Apr., 2000
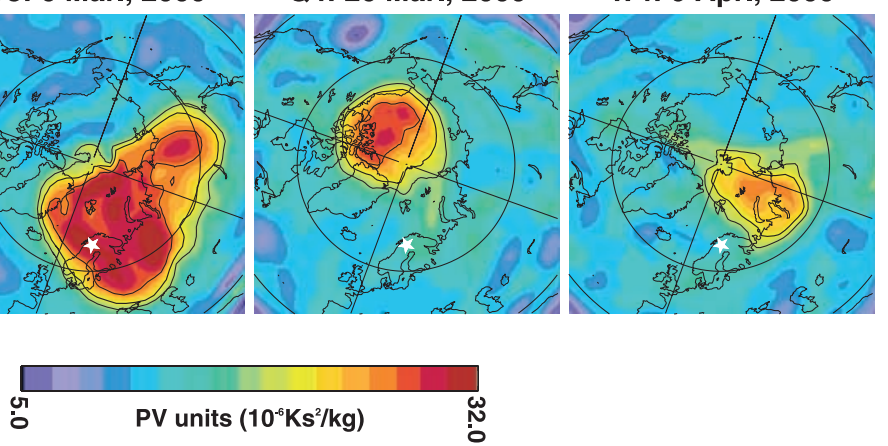

Figure 11. Potential vorticity on the $450 \mathrm{~K}$ isentropic surface.

early April (W4 in Figure 10) completely breaks up the vortex.

[80] The potential vorticity evolution in the lower stratosphere generally follows the evolution of the polar night jet. Figure 11 displays a series of potential vorticity maps on the $450 \mathrm{~K}$ isentropic surface over the course of the winter (modified potential vorticity is used herein [see Lait, 1994]). The letters in the titles of each map correspond to the letters shown in Figure 10. Both the November 23 (W1) and December $2(\mathrm{Q} 1)$ panels display the weak vortex that characterized the early lower stratospheric winter. The midDecember to early March (Q2, W2, Q3, and W3) vortex is quite strong and large, while the polar vortex in late March and early April was quite small (Q4 and W4). There are three periods of weak eddy heat flux shown in Figure 10 (Q2, Q3, and Q4). These three periods correspond to relatively symmetric polar vortices that are shown in Figure 11.

\subsubsection{Total Ozone}

[81] Total ozone in the Arctic was quite low during spring of 2000. Figure 2 displays the zonal mean total ozone obtained by TOMS. During the February-April period, a low of ozone is distinctly observed in the Arctic that is coincident with the polar vortex. Figure 12 displays the total ozone average for March over the Arctic region (updated from Newman et al. [1997]). Total ozone for March 2000 was much lower than normal.

[82] A very low ozone episode, or minihole, occurred in late November and early December 1999 over northern Europe. This episode had the lowest column ozone values ever measured by TOMS and ground-based instruments in the Northern Hemisphere during this early part of the cold season. This largely dynamical ozone reduction was observed to occur in conjunction with (early winter) polar stratospheric clouds [Hood et al., 2001; Orsolini and Limpasuvan, 2001]. While miniholes may lead to ozone loss via the formation of cold temperatures and PSCs, they are not caused by ozone loss, and they tend to occur during the winter period and are associated with upper tropospheric anticyclone systems in the storm track region of the North Atlantic [James, 1998].

\subsection{Campaign Results}

\subsubsection{Transport and Dynamics}

[83] The evolution of the vortex and polar temperatures has been broadly described herein and more precisely by Manney and Sabutis [2000] and the NOAA Climate Prediction Center's Northern Hemisphere winter summary for 1999/2000 (see http://www.cpc.ncep.noaa.gov/products/ stratosphere/winterbulletins/nh_99-00/index.html). Newman et al. [2001] quantitatively and theoretically show that the cold stratosphere during the SOLVE/THESEO 2000

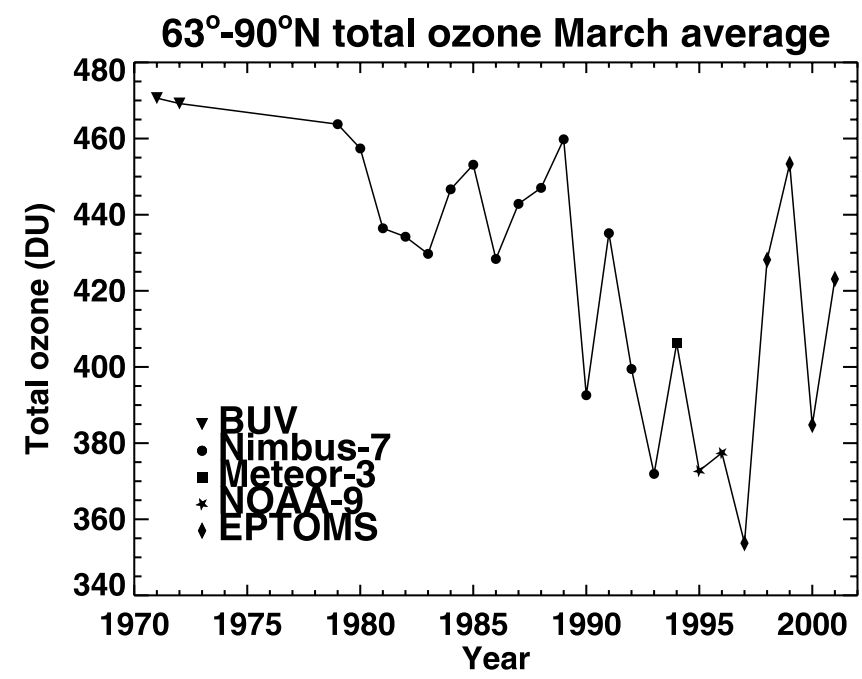

Figure 12. Total ozone averaged between $63^{\circ}$ and $90^{\circ} \mathrm{N}$ during the month of March for the Northern Hemisphere. Updated from Newman et al. [1997]. 
campaign was a result of weak tropospheric wave driving of the stratosphere. Waugh and Rong [2002] show the breakup date of the vortex as 11 April 2000. The vortex breakup and the mixing of air into the midlatitudes are modeled by Piani et al. [2002]. They show that above $420 \mathrm{~K}$ most of the air contained in the polar vortex is mixed and carried into low latitudes by the end of June, while air below $420 \mathrm{~K}$ was primarily contained in the polar region.

[84] Godin et al. [2002] describe the appearance of polar air over the OHP site in southern France. While the appearance of vortex air over OHP was relatively low in comparison with the previous 3 years, they estimate a $4 \%$ reduction of ozone in the $400-500 \mathrm{~K}$ layer as a result of low-ozone filaments over OHP. Hauchecorne et al. [2002] used a high-resolution isentropic model to estimate the transport of ozone to the midlatitudes in filamentation events coming off of the polar vortex. They found that winters with strong and cold polar vortices are associated with relatively weak filamentation compared with active years when as much as $20 \%$ of the vortex is transported to midlatitudes.

[85] The polar vortex is chemically distinct from the midlatitudes. Very large gradients of trace gas concentrations are observed in crossing the boundary of the polar vortex, forming chemical "fronts." One of the principal gases used for diagnosing the behavior of the polar vortex is nitrous oxide $\left(\mathrm{N}_{2} \mathrm{O}\right)$. Hurst et al. [2002] describe the development of a high-resolution, consistent, unified $\mathrm{N}_{2} \mathrm{O}$ data set derived from the ER-2 in situ observations. Such a data set is critical for diagnosing the evolution of the vortex and understanding tracer-tracer relationships. Greenblatt et al. [2002] use the $\mathrm{N}_{2} \mathrm{O}$ observations to describe a technique for precisely defining the edge of the polar vortex.

[86] While conventional meteorological observations and models describe the larger-scale meteorology, mesoscale models are necessary to also describe the basic thermal structure of the stratosphere. In particular, such models are necessary to describe the smaller-scale features that can lead to the formation of PSCs. Dörnbrack et al. [2002] describe the model and observations of mountain wave forced PSCs that formed in late January in the Kiruna region.

\subsubsection{Formation and Composition of PSCs}

[87] Because the winter of 1999/2000 was cold, extensive PSCs were observed over the course of the SOLVE/THESEO 2000 campaign. Using POAM III measurements, Bevilacqua et al. [2002] give a description of the extent of the PSCs during the entire campaign, and they contrast the 1999/2000 observations with previous years. In particular, PSCs were first observed by POAM in mid-November and were last observed on 15 March 2000.

[88] In situ observations of PSCs were made by both aircraft and balloons during the campaign. Fahey et al. [2001] describe the measurements of large nitric acid hydrate particles that were found over extensive portions of the polar vortex. These large nitric acid-containing particles were found at temperatures above the frost point and were large enough to considerably denitrify the stratosphere. Voigt et al. [2000] and Schreiner et al. [2002] report the first direct observations of the composition of PSCs by the Kiruna launched PSC gondola on 25 January 2001. This balloon observation showed that liquid ternary particles were seen near the frost point, solid NAT near and slightly above NAT equilibrium, and a few large-sized NAT that were in thin cloud layers below the other cloud layers. The liquid and NAT particle formation has been simulated by Larsen et al. [2002], using a detailed microphysical model to represent the simultaneously measured chemical compositions and particle sizes.

[89] B. P. Luo et al. (Large stratospheric particles observed by lidar during SOLVE/THESEO 2002 mission, submitted to Journal of Geophysical Research, 2000, hereinafter referred to as Luo et al., submitted manuscript, 2002) discuss the five types of PSCs observed by the NASA DC-8 Arotel instrument over the course of the SOLVE/THESEO 2000 campaign and derive some of the properties of these PSCs. Von König et al. [2002] use a combination of measurements from several remote sensing instruments mounted on board the DC- 8 to investigate the composition of one polar stratospheric cloud observed on 7 December 1999. A good agreement with a NAT composition is found for this PSC.

[90] As a result of the sedimentation of these large nitric acid-containing particles, extensive denitrification was observed in the polar vortex. Popp et al. [2001] report that the polar vortex had an average denitrification of $60 \%$. Denitrification was also determined by remote observations of odd nitrogen constituents [Kleinböhl et al., 2002]. Corresponding to this denitrification at $17-21 \mathrm{~km}$, Koike et al. [2002] report that slight increases in $\mathrm{NO}_{y}$ were observed from the DC- 8 at $10-12.5 \mathrm{~km}$ in March, which are interpreted as being caused by the evaporation of particles after falling from higher altitudes. While extensive denitrification was observed in the vortex, only a small dehydration was observed. In situ balloon measurements of $\mathrm{H}_{2} \mathrm{O}$ from January until March showed dehydration up to $0.5 \mathrm{ppmv}$ at 20- to 22-km altitude [Schiller et al., 2002], while ER-2 data at the highest flight levels consistently yield a dehydration of $0.2-0.3$ ppmv for the vortex as a whole [Herman et al., 2002]. The observations thus provide the first detection of a widespread, but weak dehydration in an Arctic vortex.

[91] New techniques for analyzing remotely sensed observations of PSCs have been developed and used in the SOLVE/THESEO 2000 campaign. The $t$ matrix technique utilized by Luo et al. (submitted manuscript, 2002) derives microphysical properties of PSCs using the lidar observations but assumes that the particles are spheroidal in shape. The finite difference time domain (FDTD) technique of Reichardt et al. [2002] derives microphysical properties of PSCs from lidar data assuming that the PSCs are irregular and hexagonal in shape. Strawa et al. [2002] derive a new technique for discriminating PSC type $1 \mathrm{a}$ and $1 \mathrm{~b}$ using POAM III satellite observations.

[92] The ability to model accurately the denitrification of the polar vortex by the large NAT particles is critical for future assessments of the stratosphere. A one-dimensional model of this denitrification has been developed by Jensen et al. [2002]. This study investigates the efficiency of denitrification under varying assumptions about temperature, number density, and NAD and NAT relative concentrations. Carslaw et al. [2002] simulates the evolution of the large NAT particles observed by Fahey et al. [2001] using a $3-\mathrm{D}$ model, with the motion of individual particles calculated using isentropic trajectories combined with a sedimen- 
tation velocity. The model produces NAT particles with sizes similar to those observed by Fahey et al. [2001] in the lower stratosphere, although the mechanism for their formation remains uncertain.

\subsubsection{Chemistry of the Polar Vortex}

[93] Chlorine and bromine are principally responsible for ozone loss in the polar vortex. The evolution of chlorine activation over the course of the winter was monitored by observations of $\mathrm{OClO}$ by the GOME satellite observations [Wagner et al., 2002]. These observations showed strong activation starting around 22 December, peaking in midFebruary, decreasing steeply in mid-March and ending around 22 March. The 1999/2000 winter had the highest activation level of all of the winters observed by GOME since its launch in 1995. Observation of $\mathrm{HCl}$ and $\mathrm{ClO}$ by ASUR [Bremer et al., 2002] showed relatively high levels of $\mathrm{HCl}$ in early December (first phase), with low $\mathrm{HCl}$ and high $\mathrm{ClO}$ in January (second phase), consistent with the activation on the extensive PSCs observed by POAM. In situ observations of $\mathrm{ClO}$ and $\mathrm{Cl}_{2} \mathrm{O}_{2}$ during the second phase also showed a vortex that was fully activated [R. M. Stimpfle et al., First measurements of $\mathrm{ClOOCl}$ in the stratosphere: The coupling of $\mathrm{ClOOCl}$ and $\mathrm{ClO}$ in the Arctic polar vortex, submitted to Journal of Geophysical Research, 2002.]. Satellite observations by MLS showed good agreement with these results [Santee et al., 2000; Danilin et al., 2002]. Microwave ground measurements of $\mathrm{ClO}$ profiles at Ny-Ålesund and Kiruna [Klein et al., 2002; Kopp et al., 2002] also show a highly activated vortex. This overall picture is consistent with the $\mathrm{ClO}$ observations performed on the Triple gondola which indicate that $\mathrm{ClO}$ was highly activated on 27 January and that it was still high on 1 March [B. Vogel et al., Photochemistry of $\mathrm{ClO}$ in the Arctic vortex in January and March 2000: In situ observations and model simulations, submitted to Journal of Geophysical Research, 2002]. Analysis of in situ measurements of $\mathrm{ClO}$ at sunset on the HALOZ 2 flight on 27 January 2000 suggests that the rate of recombination for $\mathrm{ClO}+\mathrm{ClO} \geq \mathrm{Cl}_{2} \mathrm{O}_{2}$ under cold stratospheric conditions is about $30 \%$ faster than currently recommended [Vömel et al., 2001].

[94] Heterogeneous reactions have been directly observed in numerous laboratory simulations, and these reactions are part of the basis for photochemical models of the stratosphere. Hanisco et al. [2002] report measurements made on the ER-2 that show air being heterogeneously processed inside a polar stratospheric cloud. Large perturbations of $\mathrm{HO}_{x}$ observations were found to be consistent with $\mathrm{HOCl}$ production via the $\mathrm{ClONO}_{2}+\mathrm{H}_{2} \mathrm{O}$ reaction.

[95] Denitrification of the polar vortex makes much less $\mathrm{NO}_{2}$ available for reacting with $\mathrm{ClO}$. Thus denitrification allows ozone loss to persist, since the remaining $\mathrm{NO}_{2}$ cannot deactivate the $\mathrm{ClO}$ catalytic loss process as effectively. Konopka et al. [2002] show that mixing of air masses with activated chlorine and air masses with high $\mathrm{NO}_{x}$ levels had only a weak influence on chlorine deactivation prior to mid-March. The deactivation is caused mainly by chemistry, i.e., $\mathrm{ClONO}_{2}$ formation with $\mathrm{NO}_{x}$ being formed from $\mathrm{HNO}_{3}$ even in denitrified air masses.

\subsubsection{Empirical and Modeled Calculations of} Chemical Ozone Loss

[96] Ozone was a fundamental observation during SOLVE/THESEO 2000. As outlined in section 2.2, satellite, balloon, ground, and aircraft observations of ozone were carried out over the entire winter of 1999/2000. In particular, while the SAGE III satellite was not launched in time for the mission, this satellite instrument will have a similar orbit and measurements as the POAM III satellite instrument. Lumpe et al. [2002] compare ozone from POAM with ER-2, MkIV, DOAS, Arotel, DIAL, and ozonesondes. This comparison shows reasonably good agreement from the tropopause to the upper stratosphere and provides confidence in the solar occultation measurements and our ability to monitor ozone with such a platform. Randall et al. [2002] use these POAM observations to construct three-dimensional ozone fields using a potential vorticity and potential temperature technique. Danilin et al. [2002] present another comparison technique that uses trajectories to show good agreement between instruments.

[97] Precise ozone loss estimates are critical components to our understanding of stratospheric photochemistry. Theory and modeling have predicted large polar ozone losses under certain conditions in the Arctic. While a number of studies revealed losses in the last few years, the loss rate uncertainties have been large, and there were unresolved assumptions in those loss estimates. Harris et al. [2002] discuss the loss estimates for a number of winters and show generally good agreement between the Match technique, vortex ozonesonde averages, MLS, HALOE, and REPROBUS (SAOZ, POAM) as long as the comparisons are precise in terms of the temporal and spatial coverage.

[98] A number of papers in this issue and previous publications calculate ozone losses for the 1999/2000 winter. Table 8 lists chemically driven ozone loss estimates derived from the SOLVE/THESEO 2000 campaign for the period from 20 January to $19 \mathrm{March}$ at about $450 \mathrm{~K}$ isentropic level. This was a period for which many techniques can provide estimates; the losses shown in Table 8 are not losses for the whole winter. The estimates shown in the table show that tremendous ozone loss occurred inside the Arctic polar vortex during the 1999/2000 winter and that there is broad agreement about the rough magnitude of the loss. However, these numbers should not be used for a detailed comparison of the loss estimates from the different techniques.

[99] The losses tend to cluster near 1.7 ppmv over this period with variations from 1.0 to 2.3 ppmv. Studies using data from instruments with coarse vertical resolution tend to have lower loss rates [e.g., Bremer et al., 2002; Kopp et al., 2002; Klein et al., 2002]. The time period of estimate is also a source of differences between analyses. The MSX ozone losses [Swartz et al., 2002] do not include the critical week in mid-March when losses were quite high, while the HALOE study period [Müller et al., 2002] begins in December. The 2.3 ppmv loss derived from MLS data is the product of the $40 \mathrm{ppbv} / \mathrm{d}$ loss rate (derived from the first two weeks of February) and the 52-day period between 20 January and 12 March with the inclusion of a vertical descent correction of $5 \mathrm{ppbv} / \mathrm{d}$. Additional sources of differences among all of the papers include descent rate differences and averaging technique. When accounted for, the losses show reasonably good consistency and provide great confidence in our ability to quantify ozone loss inside the polar vortex.

[100] These large losses are similar to model estimate ozone losses [Sinnhuber et al., 2000], with the ozone loss in 
Table 8. Polar Vortex Ozone Losses at $450 \mathrm{~K}$ on 12 March (Estimated From Observations Between 20 January and 12 March 2000)

\begin{tabular}{|c|c|c|c|}
\hline Paper & $\begin{array}{l}\mathrm{O}_{3} \text { Loss, } \\
\text { (ppmv) }\end{array}$ & $\begin{array}{l}\text { Uncertainty, } \\
\text { (ppmv) }\end{array}$ & Data Set \\
\hline $\begin{array}{l}\text { W. B. Grant et al., Use of ozone distributions, } \\
\text { potential vorticity, and cooling rates to } \\
\text { estimate Arctic polar vortex ozone loss } \\
\text { during the winter of } 1999 / 2000 \text {, submitted } \\
\text { to Journal of Geophysical Research, } 2002 \text {. }\end{array}$ & 1.5 & \pm 0.3 & LRC DIAL \\
\hline Hoppel et al. [2002] & 1.45 & \pm 0.2 & POAM III solar occultation \\
\hline Klein et al. [2002] & $0.7^{\mathrm{a}}$ & \pm 0.3 & Ny Ålesund microwave radiometer \\
\hline Kopp et al. [2002] & $\begin{array}{r}1.1^{\mathrm{a}} \\
1.4\end{array}$ & $\begin{array}{l} \pm 0.2 \\
\pm 0.6\end{array}$ & $\begin{array}{l}\text { Kiruna FTIR } \mathrm{O}_{3}, \mathrm{~N}_{2} \mathrm{O}, \mathrm{HF} \\
\text { microwave } \mathrm{O}_{3}, \text { FTIR } \mathrm{N}_{2} \mathrm{O}, \mathrm{HF}\end{array}$ \\
\hline Lait et al. [2002] & 1.7 & \pm 0.3 & PV- $\theta$ analysis - ozonesondes \\
\hline Müller et al. [2002] & $2.2^{\mathrm{b}}$ & \pm 0.3 & HALOE and OMS \\
\hline Rex et al. [2002] & 1.7 & \pm 0.2 & Match - ozonesondes \\
\hline Richard et al. [2001] & 1.8 & \pm 0.3 & ER-2 tracer-tracer \\
\hline Robinson et al. [2002] & $\begin{array}{l}1.35^{\mathrm{c}} \\
1.10^{\mathrm{d}}\end{array}$ & $\begin{array}{r} \pm 0.12 \\
\pm 0.1\end{array}$ & $\begin{array}{l}\text { DIRAC tracer-tracer } \\
\text { DESCARTES tracer-tracer }\end{array}$ \\
\hline Salawitch et al. [2002] & $\begin{array}{r}1.36^{\mathrm{e}} \\
1.76\end{array}$ & $\begin{array}{l} \pm 0.21 \\
\pm 0.21\end{array}$ & $\begin{array}{l}\text { OMS measurements } \\
\text { OMS balloon and ER-2 data }\end{array}$ \\
\hline Santee et al. [2000] & $2.3^{\mathrm{f}}$ & $\begin{array}{r}-1.21 \\
\pm 0.6\end{array}$ & UARS MLS \\
\hline Schoeberl et al. [2002] & $\begin{array}{r}1.63 \\
1.3 \\
1.54\end{array}$ & $\begin{array}{r} \pm 0.3 \\
\pm 0.1 \\
\pm 0.15\end{array}$ & $\begin{array}{l}\text { vortex avg.--ozonesondes } \\
\text { POAM III } \\
\text { forward trajectory }\end{array}$ \\
\hline Sinnhuber et al. [2000] & 1.7 & \pm 0.2 & $\begin{array}{l}\text { Ny Alesund ozonesonde, } \\
\text { SLIMCAT model }\end{array}$ \\
\hline Swartz et al. [this issue] [2002] & $1.0^{\mathrm{g}}$ & \pm 0.08 & MSX stellar occultation \\
\hline $\begin{array}{l}{ }^{\mathrm{a}} \text { Microwave and IR instruments have } \sim 10-\mathrm{k} \\
\mathrm{b} \text { Estimated between November } 1999 \text { and } 11 \\
{ }^{\mathrm{c}} \text { Estimated between } 23 \text { January and } 7 \text { March } \\
\mathrm{d} \text { Estimated between November } 1999 \text { and } 4 \\
{ }^{\mathrm{e}} \text { Estimated for } 20 \text { January to } 5 \text { March } 2000 . \\
{ }^{\mathrm{f}} \text { Loss rate }(0.04 \text { ppmv/d) estimated at } 465 \mathrm{~K} \\
\text { for downward advection. }\end{array}$ & $\begin{array}{l}\text { ical resolut } \\
\text { larch } 2001 \text {. } \\
2001 . \\
\text { n } 2 \text { and } 13\end{array}$ & 20-km altitude & days, with a 0.25 ppmv correction \\
\hline
\end{tabular}

the vortex edge region being lower than in the vortex core [Grooß et al., 2002]. Gao et al. [2001] show that the ozone loss rate is directly tied to the level of denitrification. They calculated ozone loss rates of $63 \mathrm{ppbv} / \mathrm{d}$ at $3-5 \mathrm{ppbv}$ of $\mathrm{NO}_{y}$ and $43 \mathrm{ppbv} / \mathrm{d}$ for 7-9 ppbv of $\mathrm{NO}_{y}$. Davies et al. [2002] use the SLIMCAT model to simulate both denitrification and its effect on ozone loss, and they find that a 70\% denitrification delays recovery by approximately 10 days. The CLaMS model study of Grooß et al. [2002] indicates that the effect of denitrification on ozone depletion starts in early March. In the vortex core (equivalent latitude $\geq 80^{\circ} \mathrm{N}$ ) the additional ozone depletion ascribed to denitrification is 0.13 ppmv (10\%) by 12 March and 0.26 ppmv (16\%) by 20 March.

\section{Summary}

[101] The SOLVE/THESEO 2000 campaign was the largest and most comprehensive study of the polar lower stratosphere that has ever been conducted. The 1999-2000 winter was cold, and the phase timing worked very near perfectly. The first phase occurred during this period of first appearance of temperatures cold enough for PSC formation. The second phase occurred as low temperatures covered extensive regions of the Arctic. The third phase covered the disappearance of low temperatures as the vortex began its breakup.

[102] The cold temperatures of the Arctic lower stratosphere during the 1999-2000 winter led to the formation of extensive layers of polar stratospheric clouds. While PSCs were observed during the December first phase, extensive PSCs were encountered during the second phase by the various platforms of SOLVE/THESEO 2000. These PSCs fully processed the air inside the vortex by mid-December. Temperatures tended to remain low throughout the winter and into mid-March. Again, PSCs were observed by our platforms up to mid-March.

[103] Activated chlorine levels were observed during the second and third phases of SOLVE/THESEO 2000 but were not observed in the first phase. The extensive measurements of chlorine species by a variety of instruments provide the most extensive and precise determination of the evolution of chlorine over the course of a single winter.

[104] Because of the high amounts of $\mathrm{ClO}_{\mathrm{x}}$ in the Arctic, and because of the persistence of PSCs into March, there were large chemical ozone losses in the lower stratosphere. Satellite, ozonesonde, in situ aircraft and balloon, and ground observations reveal that losses exceeded $60 \%$ in a layer of the lower stratosphere $(460 \mathrm{~K})$.

[105] Acknowledgments. We would like to thank the pilots and ground crews of all the aircraft that participated in SOLVE/THESEO 2000. In particular, we would like to thank the ER-2 pilots, James Barrilleaux, Jan V. Nystrom, and DeLewis Porter; the DC-8 pilots, Frank Batteas, William Brockett, Richard Ewers, Gordon Fullerton, and Edwin Lewis; the Swiss Air Force pilots, Oberstlt Peter Hauser and Hptm Patrik Steiner; the DLR Falcon pilots, Roland Welser and Peter Vogel; and the Mystère 20 and ARAT pilots, Christian Tardieu, Jean-Luc Pilotto, and Daniel Damien. The NASA aircraft and pilots were magnificently supported by the personnel of NASA's Dryden Flight Research Center Airborne Science Directorate, and we would like to especially thank ER-2 crew chiefs Jim Barnes and Ron Lopez and DC-8 crew chiefs Steve Davis and 
Paul Ristrim, as well as their crews. Similarly, a special mention goes to Jeannine Rüetschi and the ground support team at ELTA for the Swiss Air Force Lear jet. We thank the Swiss Air Force for providing their aircraft for this campaign. We are grateful to the Institut Geographique National for the maintenance and operation of the CNRS/INSU Mystère 20 and ARAT and to the ground support crews ably led by Noel Grand. We also acknowledge the excellent ground support provided for the DLR Falcon. Finally, we would like to thank all the staff at the Kiruna Airport and, specifically, Ulf Mukka for logistical operational support and Tommy Pettersson for his leadership. The balloon-launching teams of France's CNES and the U.S National Scientific Balloon Facility (NSBF) spent many long weeks at Esrange waiting patiently and launching efficiently. This contribution was invaluable. We would like to specifically mention and thank Pierre Faucon and his deputies Max Baron, Michel Bas, and Pierre Dedieu for their leadership of the CNES balloon-launching team from Aire sur l'Adour; Danny Ball, Dwayne Orr, and Erich Klein, the NSBF Campaign Managers of the NSBF team from Palestine, Texas, USA; and David Shiffrin of the $\mathrm{CAO}$ from Moscow for the coordination and recovery of the flights in Russia. The efforts of these visiting launch teams was most enthusiastically and ably complemented by the balloon group at Esrange, and we would particularly like to thank Borje Sjoholm, Tomas Hedquist, and Per Baldeman for their own hard work and for that of the whole launch team. Other members of the Esrange staff also contributed enormously to the success of the campaign; we would like to acknowledge Jan Englund, Stig Kemi, and Alf Wikström for their many and varied contributions and Johanna Bergstrom-Roos for her help with public relations and the organization of Commissioner Busquin's visit. Many personnel were involved at ground stations away from the main theater of operations, but their contributions are no less important. In particular, we would like to thank the individuals responsible for the operation of the ground-based instruments, for launching ozonesondes at all hours of the day and night, and for the accelerated production of data from satellites. The NASA Ames Earth Science Project Office made tremendous contributions to the operations and overall success of SOLVE. Our special thanks go to Sue Tolley, Quincy Allison, Wendy Dolci, Steve Gaines, and Joe Goosby. The shipment of the tons of science hardware to Kiruna, Sweden, could not have been accomplished without the tremendous efforts of the U.S. Air Force Material Command. Specifically, we acknowledge the efforts of Maggie Proctor and Kenneth Skiles, and our special thanks go to the men and women of the 445th at Wright Patterson AFB, the 934th of Minneapolis/St. Paul ARB, the 446th at McChord AFB, and the 452nd at March ANGB. Overflights of Russian airspace were critical to the success of SOLVE/THESEO 2000. We are especially grateful to General Valery Zakharin and his team for coordinating these over-flights. Special thanks to Academician Nicolai Laverov and Anatoli Khrenov for facilitating discussions that led to these overflights. The European Union provided financial assistance for THESEO 2000 through a number of European and national funding agencies. Acknowledgments for support are given most appropriately in all the individual research papers that are being published. Here we would like to thank DG Research of the European Commission for their overall support for THESEO 2000 through many projects in their Environment and Sustainable Development Programme and, particularly, for the coordination of THESEO 2000 through the CASSIS and CRUSOE concerted actions (ENV4CT97-0550 and EVK2-1999-00252). We would also like to thank the following national funding agencies for their important contributions and apologize to those we have inadvertently omitted: CNES, CNRS-INSU, and the PNCA Programme in France; the BMBF and Max-Planck-Gesellschaft in Germany; the National Environment Research Council and Department of Environment, Transport and the Regions in the UK; CNR and the Italian Space Agency in Italy; the Belgian Offices for Scientific, Technical and Cultural Affairs; the Norwegian Research Council; the Swedish Space Board; ETH in Switzerland; and the Finnish Meteorological Institute. SOLVE was sponsored by National Aeronautics and Space Administration through its Upper Atmosphere Research Program (UARP), the Atmospheric Effects of Aviation Project (AEAP), the Atmospheric Chemistry Modeling and Analysis Program (ACMAP), and the EOS Validation Program. This sponsorship was performed in cooperation with the National Oceanic and Atmospheric Administration (NOAA), the National Science Foundation (NSF), and the National Center for Atmospheric Research (NCAR). We would like to record our special appreciation and thanks to Kathy Wolfe and Rebecca Penkett for their dedication and effort throughout the planning, operation, and postmission analysis of SOLVE/THESEO 2000. Kathy was most ably assisted by Rose Kendall and Randy Soderholm, to whom we also send our heartfelt thanks. We are also particularly grateful to the people of Kiruna, Sweden. Their foresight led to the construction of the Arena Arctica, whose location and accommodations were major factors in the success of SOLVE/THESEO 2000. In addition, the hospitality of the residents of Kiruna made the extended visits of our numerous teams both pleasant and enjoyable. Finally, we thank the numerous persons who contributed instrument and payload descriptions, made comments on the manuscript, and corrected a number of items in our tables and figures. In particular, we thank Jochen Schreiner for the Figure 6 photo and Florence Goutail for the Figure 7 photo.

\section{References}

Barath, F. T., et al., The Upper Atmosphere Research Satellite Microwave Limb Sounder instrument, J. Geophys. Res., 98, 10,751-10,762, 1993. Becker, G., R. Müller, D. S. McKenna, M. Rex, K. S. Carslaw, and H. Oelhaf, Ozone loss rates in the Arctic stratosphere in the winter 1994/1995: Model simulations underestimate results of the match analysis, J. Geophys. Res., 105, 15,175-15,184, 2000.

Bevilacqua, R. M., M. D. Fromm, J. M. Alfred, J. H. Hornstein, G. E. Nedoluha, K. W. Hoppel, J. D. Lumpe, and C. E. Randall, Observations and analysis of polar stratospheric clouds detected by POAM III during the 1999/2000 Northern Hemisphere winter, J. Geophys. Res., 107, 8281, 10.1029/2001JD000477, 2002.

Bremer, H., M. von König, A. Kleinböhl, H. Küllmann, K. Künzi, K. Bramstedt, J. P. Burrows, K.-U. Eichmann, M. Weber, and A. P. H. Goede, Ozone depletion observed by Airborne Submillimeter Radiometer during the Arctic winter 1999/2000, J. Geophys. Res., 107, 8277, 10.1029/2001JD000546, 2002.

Burrows, J. P., M. Weber, M. Buchwitz, V. V. Rozanov, A. LadstätterWeißenmayer, A. Richter, R. DeBeek, R. Hoogen, K. Bramstedt, and K. U. Eichmann, The Global Ozone Monitoring Experiment (GOME): Mission concept and first scientific results, J. Atmos. Sci., 56, 151-175, 1999.

Carslaw, K. S., M. Wirth, A. Tsias, B. P. Luo, A. Dörnbrack, M. Leutbecher, H. Volkert, W. Renger, J. T. Bacmeister, and T. Peter, Particle microphysics and chemistry in remotely observed mountain polar stratospheric clouds, J. Geophys. Res., 103, 5785-5796, 1998.

Carslaw, K. S., J. A. Kettleborough, M. J. Northway, S. Davies, R.-S. Gao, D. W. Fahey, D. Baumgardner, M. P. Chipperfield, and A. Kleinböhl, A vortex-scale simulation of the growth and sedimentation of large nitric acid hydrate particles, J. Geophys. Res., 107, 10.1029/2001JD000467, in press, 2002

Cunnold, D. M., et al., Validation of SAGE II $\mathrm{NO}_{2}$ Measurements, J. Geophys. Res., 96, 12,913-12,925, 1991.

Danilin, M. Y., et al., Comparison of ER-2 aircraft and POAM III, MLS, and SAGE II satellite measurements during SOLVE using traditional correlative analysis and trajectory hunting technique, J. Geophys. Res., 107, 10.1029/2001JD000781, in press, 2002

Davies, S., et al., Modeling the effect of denitrification on Arctic ozone depletion during winter 1999/2000, J. Geophys. Res., 107, 10.1029/ 2001JD000445, in press, 2002

Dörnbrack, A., T. Birner, A. Fix, H. Fientje, A. Meister, H. Schmid, E. V. Browell, and M. J. Mahoney, Evidence for inertia gravity waves forming polar stratospheric clouds over Scandinavia, J. Geophys. Res., 107, 10.1029/2001JD000452, in press, 2002

Fahey, D. W., et al., The detection of large $\mathrm{HNO}_{3}$-containing particles in the winter Arctic stratosphere, Science, 291, 1026-1031, 2001.

Farman, J. C., B. G. Gardiner, and J. D. Shanklin, Large losses of total ozone in Antarctica reveal seasonal $\mathrm{ClO}_{x} / \mathrm{NO}_{x}$ interaction, Nature, 315 , 207-210, 1985

Gao, R. S., et al., Observational evidence for the role of denitrification in Arctic stratospheric ozone loss, Geophys. Res. Lett., 28, 2879-2882, 2001.

Godin, S., M. Marchand, and A. Hauchecorne, Influence of Arctic polar ozone depletion on the lower stratospheric ozone amounts at Haute-Provence Observatory $\left(44^{\circ} \mathrm{N}, 6^{\circ} \mathrm{E}\right), J$. Geophys. Res., 107, 8272, 10.1029/ 2001JD000516, 2002

Greenblatt, J. B., et al., Defining the polar vortex edge using an $\mathrm{N}_{2} \mathrm{O}$ potential temperature correlation versus the Nash criterion: A comparison, J. Geophys. Res., 107, 8268, 10.1029/2001JD000575, 2002.

Grooß, J.-U., et al., Simulation of ozone depletion in spring 2000 with the Chemical Lagrangian Model of the Stratosphere (CLaMS), J. Geophys. Res., 107, 10.1029/2001JD000456, in press, 2002.

Hanisco, T. F., et al., Quantifying the rate of heterogeneous processing in the Arctic polar vortex with in situ observations of $\mathrm{OH}, J$. Geophys. Res., 107, 8278, 10.1029/2001JD000425, 2002.

Hanson, D., and K. Mauersberger, Solubility and equilibrium vapor-pressures of $\mathrm{HCl}$ dissolved in polar stratospheric cloud materials: Ice and the trihydrate of nitric-acid, Geophys. Res. Lett., 15, 1507-1510, 1988.

Harris, N. R. P., M. Guirlet, P. A. Newman, and A. Adriani, Report on the SOLVE/THESEO 2000 science meeting, Stratos. Proc. Their Role Clim. (SPARC) Newsl., 16, 27-29, 2001

Harris, N. R. P., M. Rex, F. Goutail, B. M. Knudsen, G. L. Manney, R. Müller, and P. von der Gathen, Comparison of empirically derived ozone losses in the Arctic vortex, J. Geophys. Res., 107, 8264, 10.1029/ 2001JD000482, 2002.

Hauchecorne, A., S. Godin, M. Marchand, B. Heese, and C. Souprayen, 
Quantification of the transport of chemical constituents from the polar vortex to midlatitudes in the lower stratosphere using the high-resolution advection model MIMOSA and effective diffusivity, J. Geophys. Res., 107, 10.1029/2001JD000491, in press, 2002.

Herman, R. L., et al., Hydration, dehydration, and the total hydrogen budget of the 1999-2000 winter Arctic stratosphere, J. Geophys. Res., 107, $10.1029 / 2001001257$, in press, 2002.

Hervig, M. E., J. M. Russell III, L. L. Gordley, J. H. Park, S. R. Drayson, and T. Deshler, Validation of aerosol measurements from the Halogen Occultation Experiment, J. Geophys. Res., 101, 10,267-10,275, 1996.

Hood, L. L, B. E. Soukharev, M. Fromm, and J. P. McCormack, Origin of extreme ozone minima at middle to high northern latitudes, J. Geophys. Res., 106, 20,925-20,940, 2001.

Hoppel, K., R. M. Bevilacqua, G. Nedoluha, C. Deniel, F. Lefèvre, J. D. Lumpe, M. D. Fromm, C. E. Randall, J. E. Rosenfield, and M. Rex POAM III observations of Arctic ozone loss for the 1999/2000 winter, J. Geophys. Res., 107, 8262, 10.1029/2001JD000476, 2002.

Hurst, D. F., et al., Construction of unified, high-resolution nitrous oxide data set for ER-2 flights during SOLVE, J. Geophys. Res., 107, 8271, 10.1029/2001JD000417, 2002.

James, P. M., A climatology of ozone mini-holes over the Northern Hemisphere, Int. J. Clim., 18, 1287-1303, 1998.

Jarnot, R. F., R. E. Cofield, J. W. Waters, D. A. Flower, and G. E. Peckham, Calibration of the Microwave Limb Sounder on the Upper Atmosphere Research Satellite, J. Geophys. Res., 101, 9957-9982, 1996.

Jensen, E. J., O. B. Toon, A. Tabazadeh, and K. Drdla, Impact of polar stratospheric cloud particle composition, number density, and lifetime on denitrification, J. Geophys. Res., 107, 10.1029/2001JD000440, in press, 2002

Kilbane-Dawe, I., N. R. P. Harris, J. A. Pyle, M. Rex, A. M. Lee, and M. P. Chipperfield, A comparison of Match and 3D model ozone loss rates in the Arctic polar vortex during the winters of 1994/95 and 1995/96, J. Atmos. Chem., 39, 123-138, 2001.

Klein, U., I. Wohltmann, K. Lindner, and K. F. Künzi, Ozone depletion and chlorine activation in the Arctic winter 1999/2000 observed in Ny-Alesund, J. Geophys. Res., 107, 10.1029/2001JD000543, in press, 2002.

Kleinböhl, A., et al., Vortexwide denitrification of the Arctic polar stratosphere in Winter 1999/2000 determined by remote observations, J. Geophys. Res., 107, 10.1029/2001JD001042, in press, 2002.

Koike, M., et al., Redistribution of reactive nitrogen in the Arctic lower stratosphere in the 1999/2000 winter, J. Geophys. Res., 107, 8275 10.1029/2001JD001089, 2002.

Konopka, P., J. Grooß, G. Günther, D. McKenna, R. Müller, J. W. Elkins, D. W. Fahey, and P. J. Popp, Weak impact of mixing on the chlorine deactivation during SOLVE/THESEO 2000: Lagrangian modeling (CLaMS) versus ER-2 in situ observations, J. Geophys. Res., 107 , $10.1029 / 2001$ JD000876, in press, 2002.

Kopp, G., et al., Evolution of ozone and ozone-related species over Kiruna during the THESEO 2000 SOLVE campaign retrieved from groundbased millimeter wave and infrared observations, J. Geophys. Res., 107, 10.1029/2001JD001064, in press, 2002

Lait, L. R., An alternative form for potential vorticity, J. Atmos. Sci., 51, $1754-1759,1994$.

Lait, L. R., et al., Ozone loss from quasi-conservative Coordinate Mapping during the 1999-2000 SOLVE/THESEO 2000 campaigns, J. Geophys. Res., 107, 8274, 10.1029/2001JD000998, 2002.

Larsen, N., et al., Microphysical mesoscale simulations of polar stratospheric cloud formation constrained by in situ measurements of chemical and optical cloud properties, J. Geophys. Res., 107, 10.1029/2001JD000999, in press, 2002.

Lucke, R. L., et al., The polar ozone and Aerosol Measurement (POAM) III instrument and early validation results, J. Geophys. Res., 104, 18,785$18,799,1999$.

Lumpe, J. D., et al., Comparison of POAM III ozone measurements with correlative aircraft and balloon data during SOLVE, J. Geophys. Res., 107, 10.1029/2001JD000472, in press, 2002

Manney, G. L., and J. L. Sabutis, Development of the polar vortex in the 1999-2000 Arctic winter stratosphere, Geophys. Res. Lett., 27, 25892592,2000

McCormick, M. P., P. Hamill, T. J. Pepin, W. P. Chu, T. J. Swissler, and L. R. McMaster, Satellite studies of the stratospheric aerosol, Bull. Am. Meteorol. Soc., 60, 1038-1046, 1979.

Meilinger, S. K., T. Koop, B. P. Luo, T. Huthwelker, K. S. Carslaw, U. Krieger, P. J. Crutzen, and T. Peter, Size-dependent stratospheric droplet composition in mesoscale temperature fluctuations and their potential role in PSC freezing, Geophys. Res. Lett., 22, 3031-3034, 1995.

Moore, F. L., et al., Balloon-borne in situ gas chromatography for measurements in the troposphere and stratosphere, J. Geophys. Res., 107, 10.1029/2001JD000891, in press, 2002.

Müller, R., S. Tilmes, J. Grooß, D. S. McKenna, G. C. Toon, R. A. Stach- nik, J. J. Margitan, J. W. Elkins, and J. M. Russell III, Chemical ozone loss and chlorine activation deduced from HALOE and balloon measurements in the Arctic during the winter of 1999/2000, J. Geophys. Res., 107, 10.1029/2001JD001423, in press, 2002

Newman, P. A., Report on the SAGE III Ozone Loss and Validation Experiment, SOLVE, Stratos. Proc. Their Role Clim. (SPARC) Newsl., 15, $19-21,2000$

Newman, P. A., J. F. Gleason, R. D. McPeters, and R. S. Stolarski, Anomalously low ozone over the Arctic, Geophys. Res. Lett., 24, 2689-2692, 1997.

Newman, P. A., E. R. Nash, and J. E. Rosenfield, What controls the temperature of the Arctic stratosphere during the spring?, J. Geophys. Res., $106,19,999-20,010,2001$

Orsolini, Y. J., and V. Limpasuvan, The North Atlantic Oscillation and the occurrences of ozone miniholes, Geophys. Res. Lett., 28, 4099-4102, 2001.

Ovarlez, J., and H. Ovarlez, Water vapour and aerosol measurements during SESAME and the observation of low water vapour content layers, in Polar Stratospheric Ozone, CEC Air Pollut. Rep. 56, edited by J. A. Pyle, N. R. P. Harris, and G. T. Amanatidis, pp. 205-208, Off. for Off. Publ. of the Eur. Commun., Luxembourg, 1995

Piani, C., W. A. Norton, A. Iwi, E. A. Ray, and J. W. Elkins, Transport of ozone-depleted air on breakup of the stratospheric polar vortex in spring/ summer 2000, J. Geophys. Res., 107, 8270, 10.1029/2001JD000488, 2002

Popp, P. J., et al., Severe and extensive denitrification in the 1999-2000 Arctic winter stratosphere, Geophys. Res. Lett., 28, 2875-2878, 2001.

Randall, C. E., et al., Reconstruction of three-dimensional ozone fields using POAM III during SOLVE, J. Geophys. Res., 107, 10.1029/ 2001JD000471, in press, 2002

Reichardt, J., S. Reichardt, P. Yang, and T. J. McGee, Retrieval of polar stratospheric cloud microphysical properties from lidar measurements: Dependence on particle shape assumptions, J. Geophys. Res., 107, 10.1029/2001JD001021, in press, 2002

Rex, M., et al., Chemical ozone loss in the Arctic winter 1994/95 as determined by the match technique, J. Atmos. Chem., 32, 35-59, 1999.

Rex, M., et al., Chemical depletion of Arctic ozone in winter 1999/2000, J. Geophys. Res., 107, 8276, 10.1029/2001JD000533, 2002.

Richard, E. C., et al., Severe chemical ozone loss inside the Arctic polar vortex during winter 1999-2000 inferred from in situ airborne measurements, Geophys. Res. Lett., 28, 2197-2200, 2001.

Russell, J. M., III, L. L. Gordley, J. H. Park, S. R. Drayson, W. D. Hesketh, R. J. Cicerone, A. F. Tuck, J. E. Frederick, J. E. Harries, and P. J. Crutzen, The Halogen Occultation Experiment, J. Geophys. Res., 98, 10,77710,798, 1993.

Salawitch, R. J., et al., Chemical loss of ozone during the Arctic winter of 1999-2000: An analysis based on balloon-borne observations, J. Geophys. Res., 107, 8269, 10.1029/2001JD000620, 2002.

Santee, M. L., G. L. Manney, N. J. Livesey, and J. W. Waters, UARS Microwave Limb Sounder observations of denitrification and ozone loss in the 2000 Arctic late winter, Geophys. Res. Lett., 27, 3213-3216, 2000.

Schiller, C., A. Hofzumahaus, M. Müller, E. Klein, E.-P. Röth, and U. Schmidt, Ultraviolet actinic flux in the stratosphere: An overview of balloon-borne measurements during EASOE, 1991/92, Geophys. Res. Lett., 21, 1239-1242, 1994

Schiller, C., et al., Dehydration in the Arctic stratosphere during the SOLVE/THESE0 2000 campaigns, J. Geophys. Res., 107, 10.1029/ 2001JD000463, in press, 2002

Schoeberl, M. R., et al., An assessment of the ozone loss during the 1999/ 2000 SOLVE/THESEO 2000 Arctic campaign, J. Geophys. Res., 107, 8261, 10.1029/2001JD000412, 2002.

Schreiner, J., et al., Chemical, microphysical, and optical properties of polar stratospheric clouds, J. Geophys. Res., 107, 10.1029/2001JD000825, in press, 2002.

Shindell, D. T., D. Rind, and P. Lonergan, Increased polar stratospheric ozone losses and delayed eventual recovery owing to increasing greenhouse-gas concentrations, Nature, 392, 589-592, 1998.

Sinnhuber, B.-M., et al., Large loss of total ozone during the Arctic winter 1999/2000, Geophys. Res. Lett., 27, 3473-3476, 2000.

Strawa, A. W., K. Drdla, M. D. Fromm, R. F. Pueschel, K. Hoppel, E. V. Browell, P. Hamill, and D. Dempsey, Discriminating type Ia and Ib polar stratospheric clouds in POAM satellite data, J. Geophys. Res., 107, 10.1029/2001JD000458, in press, 2002

Swartz, W. H., J.-H. Yee, R. J. Vervack Jr., S. A. Lloyd, and P. A. Newman, Photochemical ozone loss in the Arctic as determined by MSX/UVISI stellar occultation observations during the 1999/2000 winter, J. Geophys. Res., 107, 10.1029/2001JD000933, in press, 2002.

Tsias, A., A. J. Prenni, K. S. Carslaw, T. P. Onasch, B. P. Luo, M. A. Tolbert, and T. Peter, Freezing of polar stratospheric clouds in orographically induced strong warming events, Geophys. Res. Lett., 24, 2303-2306, 1997. 
Voigt, C., et al., Nitric acid trihydrate (NAT) in polar stratospheric clouds, Science, 290, 1756-1758, 2000.

Vömel, H., D. W. Toohey, T. Deshler, and C. Kroger, Sunset observations of $\mathrm{ClO}$ in the Arctic polar vortex and implications for ozone loss, Geophys. Res. Lett., 28, 4183-4186, 2001.

von König, M., et al., Using gas phase nitric acid as an indicator of polar stratospheric clouds composition, J. Geophys. Res., 107, 8265, 10.1029/ 2001JD001041, 2002

Wagner, T., F. Wittrock, A. Richter, M. Wenig, J. P. Burrows, and U. Platt, Continuous monitoring of the high and persistent chlorine activation during the Arctic winter 1999/2000 by the GOME instrument on ERS2, J. Geophys. Res., 107, 8267, 10.1029/JD000466, 2002.

Waters, J. W., Microwave Limb Sounding, in Atmospheric Remote Sensing by Microwave Radiometry, edited by M. A. Janssen, chap. 8, John Wiley, New York, 1993.

Waters, J. W., et al., The UARS and EOS Microwave Limb Sounder (MLS) Experiments, J. Atmos. Sci., 56, 194-218, 1999.

Waugh, D. W., and P.-P. Rong, Interannual variability in the decay of lower stratospheric Arctic vortices, J. Meteorol. Soc. Jpn., in press, 2002.

Woyke, T., R. Muller, F. Stroh, D. S. McKenna, A. Engel, J. J. Margitan, M. Rex, and K. S. Carslaw, A test of our understanding of the ozone chemistry in the Arctic polar vortex based on in situ measurements of $\mathrm{ClO}, \mathrm{BrO}$, and $\mathrm{O}_{3}$ in the 1994/1995 winter, J. Geophys. Res., 104, $18,755-18,768,1999$.

Zöger, M., et al., Fast in situ stratospheric hygrometers: A new family of balloon-borne and airborne Lyman alpha photofragment fluorescence hygrometers, J. Geophys. Res., 104, 1807-1816, 1999.

A. Adriani, Consiglio Nazionale delle Ricerche, Instituto di Fisica dell'Atmosfera (CNR-IFA), Via Fosso del Cavaliere 100, I-00133 Roma, Italy. (adriani@ifa.rm.cnr.it)

G. T. Amanatidis, European Commission, Research Directorate General, 200 Rue de la Loi, SDME 7/55, B-1049 Brussels, Belgium. (georgios. amanatidis@cec.eu.int)

J. G. Anderson, Anderson Group/CCB, Department of Chemistry, Harvard University, 12 Oxford Street (Link Building), Cambridge, MA 02138, USA. (anderson@huarp.harvard.edu)

G. O. Braathen, The Norwegian Institute for Air Research (NILU), P. O.

Box 100, Instituttvein 18, N-2027 Kjeller, Norway. (geir@nilu.no)

W. H. Brune, Department of Meteorology, 503 Walker Building, Pennsylvania State University, University Park, PA 16802, USA. (brune@ ems.psu.edu)

K. Carslaw, School of the Environment, University of Leeds, Woodhouse Lane, Leeds LS2 9JT, UK. (carslaw@env.leeds.ac.uk)
M. S. Craig, Mail Stop 245-5, Ames Research Center, National Aeronautics and Space Administration, Moffett Field, CA 94035-1000, USA. (mcraig@mail.arc.nasa.gov)

P. L. Decola and M. J. Kurylo, Code YS, Headquarters, National Aeronautics and Space Administration, Washington, DC 20546-0001, USA. (pdecola@hq.nasa.gov; mkurylo@hq.nasa.gov)

M. Guirlet, ACRI-ST, 260 Route du Pin Montard, F-06904 SophiaAntipolis, France. (mag@acri-st.fr)

N. Harris, European Ozone Research Coordinating Unit, 14 Union Road, Cambridge CB2 1HE, UK. (Neil.Harris@ozone-sec.ch.cam.ac.uk)

R. S. Hipskind, Atmospheric Chemistry and Dynamics Branch, Mail Stop 245-5, Ames Research Center, National Aeronautics and Space Administration, Moffett Field, CA 94035-1000, USA. (shipskind@mail. arc.nasa.gov)

H. Küllman, Institute of Environmental Physics, University of Bremen, FB1 Postfach 330440, D-28334 Bremen, Germany. (hkuell@physik. uni-bremen.de)

N. Larsen, Middle Atmosphere Research Division, Danish Meteorological Institute (DMI), Lyngbyvej 100, DK-2100 Copenhagen, Denmark. (nl@dmi.dk)

G. Mégie, Centre National de la Recherche Scientifique, 3 rue Michel Ange, F-75794 Paris Cedex 16, France. (gerard.megie@cnrs-dir.fr)

P. A. Newman, Code 916, Goddard Space Flight Center, National Aeronautics and Space Administration, Greenbelt Road, Greenbelt, MD 20771-0001, USA. (newman@notus.gsfc.nasa.gov)

J.-P. Pommereau, Service d'Aéronomie du Centre National de la Recherche Scientifique (CNRS), BP 3, F-91371 Verrieres le Buisson Cedex, France. (pommereau@aerov.jussieu.fr)

L. R. Poole, Mail Stop 435, Radiation and Aerosols Branch, Langley Research Center, National Aeronautics and Space Administration, Hampton, VA 23681-2199, USA. (1.r.poole@larc.nasa.gov)

M. R. Schoeberl, Code 910, Goddard Space Flight Center, National Aeronautics and Space Administration, Greenbelt Road, Greenbelt, MD 20771-0001, USA. (schom@hyperion.gsfc.nasa.gov)

F. Stroh, Institut für Stratosphärische Chemie, ICG-1, Forschungszentrum Jülich GmbH, Leo Brandt Str. No. 1, D-52425 Jülich, Germany. (f.stroh@ fz-juelich.de)

O. B. Toon, Laboratory for Atmospheric and Space Physics, Program in Atmospheric and Oceanic Science, Campus Box 392, University of Colorado, Boulder, CO 80309-0392, USA. (toon@lasp.colorado.edu)

C. R. Trepte, Mail Stop 475, Radiation and Aerosols Branch, Langley Research Center, National Aeronautics and Space Administration, Hampton, VA 23681-2199, USA. (c.r.trepte@larc.nasa.gov)

M. Van Roozendael, Belgian Institute for Space Aeronomy (BIRA-IASB), Ringlaan-3-Avenue Circulaire, B-1180 Brussels, Belgium. (michelv@ oma.be) 